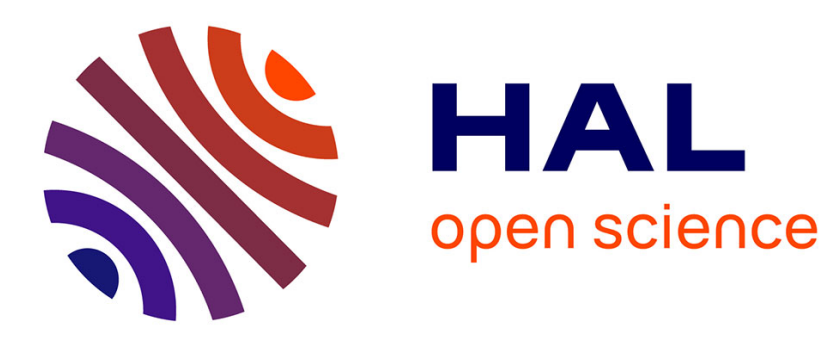

\title{
Optimization of metabolite detection by quantum mechanics simulations in magnetic resonance spectroscopy \\ Giulio Gambarota
}

\section{- To cite this version:}

Giulio Gambarota. Optimization of metabolite detection by quantum mechanics simulations in magnetic resonance spectroscopy. Analytical Biochemistry, 2017, 529, pp.65-78. 10.1016/j.ab.2016.08.019 . hal-01361778

\section{HAL Id: hal-01361778 \\ https://hal.science/hal-01361778}

Submitted on 12 Sep 2016

HAL is a multi-disciplinary open access archive for the deposit and dissemination of scientific research documents, whether they are published or not. The documents may come from teaching and research institutions in France or abroad, or from public or private research centers.
L'archive ouverte pluridisciplinaire HAL, est destinée au dépôt et à la diffusion de documents scientifiques de niveau recherche, publiés ou non, émanant des établissements d'enseignement et de recherche français ou étrangers, des laboratoires publics ou privés. 
Optimization of metabolite detection by quantum mechanics simulations in magnetic resonance spectroscopy

\author{
Giulio Gambarota ${ }^{1,2}$ \\ ${ }^{1}$ Université de Rennes 1, LTSI, Rennes, F-35000, France \\ 2 INSERM, UMR 1099, Rennes, F-35000, France
}

Running Head: Quantum mechanics simulations in magnetic resonance spectroscopy

Corresponding author:

Giulio Gambarota, Ph.D.

Professor

INSERM, U1099

Université de Rennes 1, LTSI

35043 Rennes, France

tel: 0223234849

email: gambarota@gmail.com

Word count: $\mathrm{xxxx}$

Number of figures and tables: 6

Number of references: 120 


\begin{abstract}
Magnetic resonance spectroscopy (MRS) is a well established modality for investigating tissue metabolism in vivo. In recent years, many efforts by the scientific community have been directed towards the improvement of metabolite detection and quantitation.

Quantum mechanics simulations allow for investigations of the MR signal behaviour of metabolites; thus, they provide an essential tool in the optimization of metabolite detection.

In this review, we will examine quantum mechanics simulations based on the density matrix formalism. The density matrix was introduced by von Neumann in 1927 to take into account statistical effects within the theory of quantum mechanics. We will discuss the main steps of the density matrix simulation of an arbitrary spin system and show some examples for the strongly coupled two spin system.
\end{abstract}

Keywords: Keywords: Quantum Mechanics simulations; Density Matrix; Magnetic Resonance Spectroscopy; Metabolites; Strongly coupled spin systems. 


\section{Introduction}

Nuclear magnetic resonance (NMR) is a Janus-faced physical phenomenon. Janus was a Roman god, with no equivalent in the Greek mythology. He was the god of the beginnings the first month of the year, January, was named after him [1]. Janus was typically depicted with two faces (Figure 1). Similarly to this Roman god, the NMR phenomenon has two diametrically opposed faces. Since the very beginning, at the time of its discovery NMR was described in two completely different ways: Edward Purcell, at Harvard, pictured the NMR as quantum transitions between energy levels whereas Felix Bloch, at Stanford, viewed the NMR as the precession of a magnetization vector obeying the laws of electromagnetism [2-4]. Thus, two diametrically opposed views to describe the same phenomenon: quantum mechanics on one hand, classical physics on the other hand. An excellent account of the NMR discovery can be found in the article entitled "Quantum states and precession: The two discoveries of NMR" written by John Ridgen [5].

Within the historical context of the NMR discovery [6, 7] we cannot avoid to mention the lesser-known NMR pioneer Cornelius Gorter and his "ingenious early attempts" (as Bloch later called them [8]) to observe the magnetic resonance phenomenon [9, 10]. The Dutch physicist tried first in 1936 to detect MR in LiF using a calorimetric method, in order to measure the heating caused by energy absorption at the resonance condition. Then, he tried in 1942 to observe the resonance employing a dispersion method; again with little luck. With respect to the unsuccessful experiments of Gorter, Van Vleck later recalled: "The real difficulty arose from the fact that he used too much power and too pure materials, things that are advantages in most physical experiments [...] If Gorter had tried to detect the resonance in ordinary tap water [...] he might have succeeded." [11].

In this review we will focus on the quantum mechanics viewpoint of the magnetic resonance phenomenon. We will examine the role of quantum mechanics -and more precisely the density matrix formalism- in the optimization of metabolite detection in vivo by magnetic resonance spectroscopy (MRS). Measurements of metabolites in vivo present a number of challenges, mainly related to 1) the intrinsically low-sensitivity of MRS and 2) the spectral overlap among resonances of different metabolites. Quantum mechanics simulations allows for investigations of the MR signal behaviour of metabolites. These simulations aid in 1) identifying the optimal acquisition parameters for in vivo measurements and 2) developing novel acquisition schemes that could further improve metabolite detection and quantification. 


\section{Magnetic resonance spectroscopy}

Magnetic resonance spectroscopy is a well established modality for investigating tissue metabolism in vivo. ${ }^{1} \mathrm{H}$ MR spectra can be routinely obtained in clinical settings with MRI systems operating at the field strength of $1.5 \mathrm{~T}$ and $3 \mathrm{~T}$, using standard equipment. In the next paragraphs we will briefly review the most common applications of ${ }^{1} \mathrm{H}$ MRS in clinical and research scenarios -cautiously avoiding the perennial question regarding the clinical utility of ${ }^{1} \mathrm{H}$ MRS [12-16]. The objective here is to identify the specific cases (which organ/tissue? which metabolite?) where the quantum mechanics approach is necessary.

Nowadays in vivo ${ }^{1} \mathrm{H}$ MRS is mostly performed on brain, prostate, muscle, liver and breast. Undoubtedly, brain MR spectra display the largest number of metabolites compared to any other organ/tissue. The major metabolites in brain include $\mathrm{N}$-acetylaspartate, creatine, choline, glutamate, glutamine, myo-inositol, taurine, GSH and GABA [13, 14]. A considerable overlap among the resonances of these metabolites is present. A number of editing methods were originally proposed, more than 20 years ago, with the focus on the quantification of GABA [17-20]; the investigation of new methods to improve quantification of GABA (and other brain metabolites) is still a current object of research [21]. In general, the driving force has been the investigation of brain function and metabolism [22-24], also with specific interest to study efficacy of therapy, as in the case of GABA tissue levels in patients with epilepsy, for instance $[18,25]$. At the field strength of clinical MRI systems, many brain metabolites (glutamate, glutamine, myo-inositol, etc. [26]) behave as strongly coupled spin systems -we will see the exact definition of 'strongly coupled' later on. At this stage we can say that the quantum mechanics approach is needed to simulate the signal behavior of these "particular" spin systems; in this context, the density matrix formalism has been essential in the optimization of a number of metabolites present in brain.

Another organ that has attracted the attention of MR spectroscopists from the very beginning of clinical MRS applications is the prostate, for the diagnosis of prostate cancer [27, 28]. The ${ }^{1} \mathrm{H}$ MR spectrum of healthy prostate tissue reveals the resonances of citrate and choline; a reduction in citrate and an increase in choline is observed in cancer tissue. As in the case of certain brain metabolites, citrate requires quantum mechanics modelling to precisely simulate the MR signal in order to determine the optimal pulse sequence and pulse sequence parameters $[29,30]$.

The ${ }^{1} \mathrm{H}$ MR spectrum of muscle tissue includes resonances of choline-containing compounds, creatine, taurine and two groups of lipid signals: the intramyocellular (IMCL) and 
extramyocellular (EMCL) lipids $[31,32]$. There is a frequency shift between the IMCL and EMCL, due to the magnetic susceptibility difference between the spherical and cylindrical compartments of the IMCL and EMCL. None of the main metabolites typically found in muscle requires the quantum mechanics approach for proper detection. Same conclusion applies to the MR spectrum of liver tissue, which includes resonances of choline-containing compounds and the resonances of the intrahepatocellular lipids [33, 34]. It should be pointed out that taurine (present in muscle) is a strongly coupled spin system; however, it could be detected without the need of quantum mechanics simulations.

In MRS of breast, similarly to the case of prostate, the main focus has been on the diagnosis of cancer, with choline-containing compounds being the biomarker of interest [35-37]. Recently, the role of lipids in breast cancer has also attracted the attention of researchers [38] and a number of ${ }^{1} \mathrm{H}$ MRS studies have been dedicated to the measurement of breast adipose tissue composition, as a potential cancer biomarker [39-41]. Overall, for the detection of the main metabolite of interest in breast MRS (choline) there is no need of quantum mechanics simulations.

Given the growing interest in the measurement of lipid composition in vivo, a quick clarification on quantum mechanics simulations applied to lipid resonances is necessary. The long lipid hydrocarbon chains are substantially more complex than the smaller molecules of metabolites. Some moieties of the hydrocarbon chains behave as strongly coupled spin systems, and thus quantum mechanics simulations are required for investigations of the MR signal of lipid resonances. Only a handful of studies have been dedicated to this subject [4245]. To reduce the complexity of the problem, a simplified model with only a subset of spins (seven or ten) was considered in the simulations. Due to the lack of precise knowledge of the spin system parameters and relaxation effects, simulations of lipid resonances have been to date only partially successful. 


\section{Quantum mechanics}

Quantum mechanics was developed during the first quarter of the twentieth century to investigate and explain a number of phenomena occuring on the time and spatial scale of atomic processes [46]. One of these phenomena was the black-body radiation, which couldn't be explained by the laws of classical physics. The solution to this problem came from Max Planck. In 1900 he put forward the hypothesis of the "quantum of action": the energy is quantized, that is, it appears in discrete, quantized values; this idea could be considered as the beginning of quantum mechanics. In a letter to the american physicist Robert Wood, Planck referred to this hypothesis as a "desperate hypothesis" [47, 48].

Another milestone was set with the investigation of the photoelectric effect; similarly to the case of the black-body radiation, classical physics failed to explain this phenomenon. In his annus mirabilis (1905) Albert Einstein provided the solution by introducing the "quantum of light". Among the last milestones of quantum mechanics was the development of the wave equation by Schroedinger in 1927:

$$
i \hbar \frac{d \psi}{d t}=H \psi
$$

where $\psi$ is the wavefunction, $H$ is the Hamiltonian, $\hbar$ the Planck constant. The wave formulation of quantum mechanics stated that a particle in the microscopic world can be described by a wavefunction, which represents the quantum state of the particle and obeys the Schroedinger equation; the Hamiltonian contains all the information related to the energy interactions that characterize the given system. A simple example is the so-called particle in a box (that is, the model of an eletron in a potential well): by solving the Schroedinger equation we obtain the wavefunction and all the physical quantities characteristic of the particle in this scenario.

Two atypical meetings are noteworthy in the development of quantum mechanics: the Solvay Conferences of 1911 and 1927. These conferences were organized by Ernest Solvay, a Belgian chemist and philanthropist who became rich with the revenues from his patent on the ammonia-soda process (or Solvay process); he decided to organize a series of meetings where scientists could share and discuss scientific results and ideas. The participation at these meetings was restricted to a limited number of invited scientists. The first Solvay Conference, entitled "La théorie du rayonnement et les quanta", took place in 1911 when the overall formalism of the quantum mechanics was still at its beginning. The first words of the Conference Proceedings (in French [49]) read: "Un Conseil scientifique (sorte de Congrès privé) s'est réuni à Bruxelles, sous les auspices de M. Ernest Solvay, du 30 octobre au 3 
novembre 1911, dans le but de discuter une série de points controversés des théories physiques modernes." Thus, the first Solvay Conference was "a kind of private congress" that took place in Bruxelles "with the objective to discuss a number of controversial issues of the modern physics theory". In the opening speech, Hendrik Lorentz referred to "the desperate hypothesis" of Planck as "the beautiful hypothesis": "[...] la belle hypothèse des éléments d'énergie, émise pour la première fois par M. Planck et appliquée à de nombreux phénomènes par M. Einstein, M. Nernst et d'autres, a été un précieux trait de lumière. Elle nous a ouvert des perspectives inattendues et même ceux qui la regardent avec une certaine méfiance doivent reconnaître son importance et sa fécondité. Elle mérite donc bien d'ètre le sujet principal de nos discussions [...]'. Lorentz highlighted the relevance of Planck's idea, "this sudden illumination", when stating that "even those who are skeptical about this hypothesis should recognize its importance. This hypothesis truly deserves to be the main subject of our discussion [...]".

By 1927, the year of the fifth Solvay Conference entitled "Électrons et photons", quantum mechanics had reached a mature state with the development of the wave mechanics and Schroedinger equation. Niels Bohr, Max Born, William Lawrence Bragg, Léon Brillouin, Arthur Compton, Marie Curie, Louis de Broglie, Peter Debye, Paul Dirac, Paul Ehrenfest, Albert Einstein, Werner Heisenberg, Hendrik Kramers, Paul Langevin, Hendrik Lorentz, Wolfgang Pauli, Max Planck and Erwin Schroedinger were among the participants of this meeting [50]. It is hard to think of any other meeting in the history of science that could compare to this one, in terms of concentration of geniuses.

In the introductory courses of quantum mechanics we learn to solve the Schroedinger equation for different scenarios, in order to obtain the wavefunction and calculate the physical quantities of interest in a given physical system. Here we need to take a step further. In the field of MRS we are interested in the magnetic properties of a particle, specifically the magnetization (spin) of the hydrogen atom nuclei (i.e., protons). Certainly, the quantum state of a spin is also described by a wavefunction; on the other hand, to simulate the MR signal we need to introduce a new, additional concept since the wavefunction alone is not sufficient for achieving our goal. This new concept is the so-called density matrix; now, why do we need the density matrix? The signal in an MRS experiment originates from many nuclei of hydrogen atoms, that is, from an ensemble of spins. In general, when we investigate the behaviour of an ensemble of particles and we do not have the exact knowledge of the state of each individual particle, we need to use statistical approaches. This occurs in classical physics 
as well as in quantum mechanics. In the case of phenomena which obey the laws of classical physics, we employ the classical statistical mechanics to investigate the physical properties of the ensemble. Likewise, in the case of quantum mechanics phenomena, we need to enter the field of quantum statistical mechanics to combine the quantum mechanics with the statistical approach. The density matrix was introduced by von Neumann in 1927, with Dirac further developing this concept in 1929, to take into account statistical effects/phenomena within the theory of quantum mechanics $[51,52]$. The density matrix contains all the statistical information about the status of the ensemble. It should be pointed out that the density matrix is actually a quantum mechanical operator, thus the proper nomenclature is "density operator"; on the other hand, it is typically referred to as density matrix, since its most common representation is in a matrix form.

In the following paragraphs, we will introduce the mathematical definition of the density matrix and the equation that governs the time evolution of the density matrix. We will use the Dirac notation, that is, the symbols " < " and " | > which are called "bra" and "ket", respectively; the bra is the conjugate transpose of the ket. These symbols were introduced by Dirac (with a bit of humor in choosing the names bra-ket) to represent generic vectors; in the quantum mechanics context, these vectors are the wavefunctions which describe the quantum states.

In general, a spin system can be in quantum state $|\Psi\rangle$ that is a superposition of basis functions (eigenstates); this quantum state can therefore be written as a linear combination of eigenstates $\mid \varphi_{n}>$ with coefficients $c_{n}$ :

$$
\left|\Psi>=\sum_{n} c_{n}\right| \varphi_{n}>
$$

and in this case, the system is said to be in a pure state $|\Psi\rangle$. The expectation value (i.e., mean value) of a physical quantity $A$ (described by the quantum operator denoted $A$ ) is given by the formula:

$$
<A>=<\Psi|A| \Psi>=\sum_{n n^{\prime}} A_{n n^{\prime}} c_{n}^{*} c_{n^{\prime}}
$$

where $A_{n n^{\prime}}$ are the elements of the matrix that represents the operator $A$.

When considering an ensemble of spins, the quantum state of the ensemble could consist of spins in different pure states, $\left|\Psi_{1}\right\rangle,\left|\Psi_{2}\right\rangle, \ldots,\left|\Psi_{i}\right\rangle$, with each state having a given statistical weight $p_{i}$. In this situation, the systems is said to be in a mixed state $|\Phi\rangle$ and this quantum state is written as: 


$$
\left|\Phi>=\sum_{i} p_{i}\right| \Psi_{i}>
$$

In the scenario of a mixed state, we can calculate the expectation value of a physical quantity $A$ by taking into consideration the different states and their statistical weight $p_{i}$ :

$$
<A>=<\Phi|A| \Phi>=\sum_{i} p_{i}<A>_{i}=\sum_{i} p_{i} \sum_{n n^{\prime}} A_{n n^{\prime}} c_{n i}^{*} c_{n^{\prime} i}=\sum_{n n^{\prime}} A_{n n^{\prime}} \sum_{i} p_{i} c_{n i}^{*} c_{n^{\prime} i}
$$

Now, a square matrix $\sigma$-the density matrix- can be defined and the density matrix elements are equal to:

$$
\sigma_{n n^{\prime}}=\sum_{i} p_{i} c_{n i}^{*} c_{n^{\prime} i}
$$

As a consequence, the density matrix operator can be written as follows:

$$
\sigma=\sum_{i} p_{i}\left|\Psi_{i}><\Psi_{i}\right|
$$

Using the relations above, the expectation value of physical quantity $A$ is:

$$
<A>=\sum_{n n^{\prime}} A_{n n^{\prime}} \sum_{i} p_{i} c_{n i}^{*} c_{n^{\prime} i}=\sum_{n n^{\prime}} A_{n n^{\prime}} \sigma_{n n^{\prime}}=\operatorname{Tr}(A \sigma)
$$

where "Tr" represents the trace operation. Thus, from the knowledge of the density matrix it is possible to determine the value of any physical variable $A$ of the system using the above relation, i.e., by calculating the trace of the product of the density matrix and the operator corresponding to the physical quantity of interest.

The elements of density matrix have the following physical meaning: the diagonal element $\sigma_{i i}$ represent the probability that the system is in the state $\left|\Psi_{i}\right\rangle$; that is, $\sigma_{i i}$ provides the population of spins in the state $\left|\Psi_{i}\right\rangle$. The off-diagonal elements $\sigma_{i j}$ are terms that connect two different states $\left|\Psi_{i}\right\rangle$ and $\left|\Psi_{j}\right\rangle$; these off-diagonal elements are the so-called coherences of the states $\left|\Psi_{i}\right\rangle$ and $\left|\Psi_{j}\right\rangle$.

Starting from the Schroedinger equation, it is possible to derive the equation that governs the time evolution of the density operator, the Liouville - von Neumann equation:

$$
i \hbar \frac{d \sigma(t)}{d t}=[H(t), \sigma(t)]
$$

In this equation, the square-bracket symbol "[ ]" represents the so-called commutator symbol defined as $[\sigma, H]=\sigma H-H \sigma$. The Liouville - von Neumann equation for the density matrix is the equivalent of the Schroedinger equation for the wavefunction. The solution of the Liouville - von Neumann equation for a time-independent Hamiltonian is:

$$
\sigma(t)=e^{-i t H} \sigma(0) e^{i t H}
$$


where $\sigma(0)$ is the density matrix at time $=0$ and $\sigma(t)$ is the density matrix at time $t$. Thus this formula allows us to evaluate the time evolution of the density matrix, once the initial density matrix and the Hamiltonian are known.

Finally, it should be noted that the density matrix is the quantum mechanical analogue to the classical ensemble distribution function, in the sense that it can be used in the same way as the classical distribution function to define the average values of any physical quantity. In the classical case, these averages are weighted means; in quantum mechanics these average are defined as the trace of the product of the density matrix and the operator of interest. In the section Density matrix simulations we will provide some examples of application of the density matrix to the MR signal simulations. In the next section we will consider the classification of spin systems into weakly coupled and strongly coupled systems. 


\section{Weakly coupled and strongly coupled spin systems}

In order to simulate the MR signal behaviour of a given metabolite, we need to know the proton chemical shifts and $J$ coupling constants. First, we will examine the physical phenomenon behind the chemical shifts and $J$ coupling constants. Protons of different hydrogen atoms in a metabolite might precess at different frequencies (chemical shifts), as the different protons might experience different local magnetic fields. The origin of the chemical shift is related to the fact that the applied static magnetic field Bo will induce electron currents in the molecule; these currents produce local magnetic fields which are on the order of $10^{-6}$ times the external field, that is, we deal with resonance frequency differences of parts per million (ppm). In addition, proton MR spectra of many metabolites display a structure which cannot be explained only in terms of chemical shifts within molecules. The additional splitting of the MR peaks from each proton into multiplets is related to the (indirect) interaction between nuclei via the bonding electrons. The difference between any two adjacent components of a multiplet is equal to the value of the $J$ coupling constant.

Once the chemical shifts and $J$ coupling constants of a given metabolite are known, it is possible to determine whether the metabolite should be modelled as a 'weakly' or 'strongly' coupled spin system, at a given field strength. The distinction between the weakly and strongly coupled systems is based on the following criterion: when the difference between the chemical shifts is much larger than the $J$ coupling constant, the spin system is weakly coupled. In other words, two spins with chemical shifts of $v_{1}$ and $v_{2}$ and a $J$ coupling constant equal to $J_{12}$ are weakly coupled if $\left|v_{1}-v_{2}\right| \gg J_{12}$. A typical example of a weakly coupled spin system is lactate [26]: the $\mathrm{CH}_{3}$ protons resonate at $1.3 \mathrm{ppm}$ and the $\mathrm{CH}$ protons resonate at 4.1 ppm, with the $J$ coupling constant being $7 \mathrm{~Hz}$. At $1.5 \mathrm{~T}$, the chemical shift difference between the $\mathrm{CH}_{3}$ and $\mathrm{CH}$ protons is $\sim 180 \mathrm{~Hz} \gg 7 \mathrm{~Hz}$, so the spin system of lactate can be modelled as a weakly coupled system. When the difference between the chemical shifts is not much larger than the $J$ coupling constant, that is, the criterion $\left|v_{1}-v_{2}\right| \gg J_{12}$ is not satified, the spin system is strongly coupled. A typical example of a strongly coupled system is citrate. The citrate protons resonate at two frequencies with the difference being $0.146 \mathrm{ppm}$ and the $J$ coupling constant is $15 \mathrm{~Hz}$. It should be pointed out that different values of chemical shifts and $J$ coupling constant of citrate could be found in the literature; it has been shown that these values change with cation concentration and $\mathrm{pH}$ [53]. At $1.5 \mathrm{~T}$ the value of the chemical shift difference $\delta$ is $9 \mathrm{~Hz}$, which is not larger than $J$ coupling constant; as a consequence at $1.5 \mathrm{~T}$ we need to model citrate as a strongly coupled spin system. Same argument applies at the 
field strength of $3 \mathrm{~T}$ : the chemical shift difference $(\delta=18 \mathrm{~Hz})$ is not much larger than the $J$ coupling constant $(15 \mathrm{~Hz}$, as the $J$ coupling constant value is independent of the magnetic field strength). Now we examine the case of citrate at the field strength of $11.7 \mathrm{~T}$. The chemical shift difference is $\delta=70 \mathrm{~Hz}$, which is certainly larger than the $J$ coupling constant. However, despite the fact that $\delta$ is more than four times the $J$ coupling constant, we still cannot classify citrate as a weakly coupled spin system. Indeed, it has been shown that even at 11.7 $\mathrm{T}$ the MR signal of citrate display a signal behaviour (i.e., coherence transfer effects during interpulse delays) which could occur only in strongly coupled spin systems [54]. Thus, with respect to the criterion of weakly vs strongly coupled, one should pay attention to the fact that a spin system is weakly coupled only when the difference between the chemical shifts is much larger than the $J$ coupling constants. In all other cases, the spin system should be classified as strongly coupled.

Why is this classification 'weakly vs strongly coupled' important? It is important because in the case of weakly coupled spin systems it is possible to use the approach of the product operator formalism [55, 56], which was introduced in 1983 as a simplification of the fully quantum-mechanics formalism of the density matrix. The product operator formalism is a convenient description for weakly coupled spin systems: it introduces simple rules to account for the effects of chemical-shifts, $J$ coupling and radiofrequency pulses. The product operator formalism is a rigorous method to calculate the signal of spin systems; its drawback, however, is that it is valid only for weakly coupled spin systems. Thus, when dealing with strongly coupled spin systems, we need to resort to quantum mechanics tool introduced by von Neumann in 1927, i.e., the density matrix. Finally, one should also note that in addition to weakly vs strongly coupled spin systems, some spins in a number of metabolites behave as singlets (that is, uncoupled spins); in this case, the Bloch equations could be used to simulate the signal behaviour. For instance the $\mathrm{CH}_{2}$ protons of glycine, which yield a singlet at 3.55 ppm, are an example of uncoupled spins [26]. 


\section{The need of MR signal simulations for in vivo MRS}

The challenges of in vivo ${ }^{1} \mathrm{H}$ MRS are related to the low tissue levels of metabolites $(\sim \mathrm{mM}$, "low" relative to the sensitivity of MRS), the limited amount of time available for in vivo experiments and the spectral overlap among resonances. Even at a high field strength ( $7 \mathrm{~T}$ for MR research systems dedicated to human studies) spectral overlap still hampers the quantification of a number of metabolites.

Many different approaches have been proposed throughout the years for optimizing metabolite detection. From a pragmatic point of view, these approaches can be classified in two main groups: 1) approaches dedicated to the parameter optimization of the STEAM (stimulated echo acquisition mode) and PRESS (double spin-echo point resolved spectroscopy) sequences [57-81], the two commonly used pulse sequences that are readily available on clinical scanners and 2) approaches focused on the development of new pulse sequences; some examples are provided in [17-21, 82-93].

Another way of classifying the different optimization methods is based on the spin system characteristics of the metabolite of interest: that is, whether the metabolite of interest is represented by a coupled or uncoupled spin system. When the metabolite of interest has coupled resonances with the overlapping (confounding) resonance being a singlet -such as the GABA resonance underneath the singlet of creatine, for instance- a number of methods (based on interpulse-delay optimization, difference spectroscopy editing, multiple quantum coherence editing, etc) have been implemented in vivo, see for instance [57-93]. A different situation occurs when the metabolite of interest is a singlet and the overlapping resonance is a coupled spin system -for instance the glycine singlet resonance underneath the myo-inositol. In this case, one option is to use a long echo time (or multiple echo times) in order to minimize the MR signal of the coupled resonance, by exploiting the intrinsic $J$ couplinginduced dephasing [94-96]. Another option is to simulate the $J$ modulation of the coupled resonance and choose a relatively short echo time that minimizes the signal intensity of the coupled resonance, see for instance [97, 98]; in the latter option, the losses due to T2 relaxation are also minimized.

In general, regardless of the specific approach implemented to quantify a given metabolite, it is essential to perform signal simulations to determine the optimal pulse sequence and sequence parameters. The density matrix formalism allows for the determination of the MR signal of spin systems, including the strongly coupled systems. The analytical calculations using the density matrix are very cumbersome even for a strongly coupled two-spin system. 
Examples of these calculations are provided in a number of studies [29, 30, 54, 99-101]. In particular, for the two-spin system it is still fairly feasible to account analytically for the effects of the $180^{\circ}$ pulses, as in the PRESS sequence. The calculation for the STEAM sequence on the other hand is quite prohibitive [30] since the $90^{\circ}$ pulses and magnetic field gradients exacerbate the already complicated nature of the analytical approach. In spin systems with three or more spins, analytical calculations would easily take months of work. Thus, it is crucial to adopt the approach of computer simulations to obtain the MR signal of a strongly coupled spin system. In the next section, we will consider in detail the density matrix simulations of strongly coupled spins systems. 


\section{Density matrix simulations}

\section{Theory}

There are a number of books [102-107] and articles [108-117] with a detailed description of the density matrix formalism applied to MR spectroscopy problems. The goal here is to guide the reader through the main steps of the density matrix simulations, providing the general guidelines, a certain insight and some details. We start with the simulation of a strongly coupled two-spin system. This specific case was among the first applications of the density matrix approach to in vivo MRS: it was used for optimizing the detection of citrate, metabolite of interest in the diagnosis of prostate cancer. The strongly coupled two-spin system also represents the ideal spin-system prototype to introduce the basic concepts of density matrix simulations: indeed, it is the simplest spin system that contains all the necessary ingredients, i.e., the chemical shift and $J$ coupling constant. The notation often adopted to indicate each spin uses the letters of the alphabet: two strongly coupled spins are denoted by letters that are close to each other in the alphabet (A and B, for instance); whereas two spins that are weakly coupled are denoted by letters that are far from each other (AX spin system). For instance, the spin system of citrate -which can be modelled as a strongly coupled two-spin system- is called an $\mathrm{AB}$ spin system, whereas for lactate the spin system is an $\mathrm{AX}_{3}$ system.

The first step consists in evaluating the Hamiltonian of the spin system. As we said previously, a quantum system is defined by its Hamiltonian, which contains all the energy interactions of the given system. In order to write down the Hamiltonian we need to know the values of the chemical shifts and $J$ coupling constants. For an AB spin system, we denote $\omega_{A}$ and $\omega_{B}$ the chemical shifts of the spin A and B, respectively, and $J_{A B}$ the coupling constant. We use the angular frequency units for the Hamiltonian parameters, $\omega$ and $J$, so that we can write the Hamiltonian in angular frequency units, with the Planck constant set equal to unity; furthermore, since we restrict our discussion to the ${ }^{1} \mathrm{H}$ MRS, we deal only with spins having quantum number of $1 / 2$.

The energy interactions of a coupled two-spin system are: 1) the Zeeman interaction, which represents the interaction between the spins and the applied magnetic field and 2) the $J$ coupling interaction, which accounts for the electron-mediated interaction between the (nuclear) spins. The $J$ coupling interaction is also called "indirect coupling" to distinguish it from the "direct", dipole-dipole interaction between spins. Here we can neglect the dipolar 
interaction, since it averages to zero under the assumption of fast isotropic molecular reorientation.

The Zeeman interaction is written as the product of the chemical shift and the z-component of spin angular momentum operator (the static magnetic field is applied, as usually, along the $\mathrm{z}$ direction). The $J$ coupling interaction is equal to the scalar product of the angular momentum operators of the two spins -this is the reason why the $J$ coupling interaction is often referred to as "scalar" coupling. Historically, the angular momentum operators of a two-spin system (the A and B spins in this case) have been denoted by the letter $I$ and $S$. Now we can write the Hamiltonian:

$$
H=\omega_{A} I_{z}+\omega_{B} S_{z}+J_{A B} I \cdot S
$$

where to summarize $\omega_{A}$ and $\omega_{B}$ are the chemical shifts of the spin $A$ and $B$, respectively, $J_{A B}$ is the $J$ coupling constant, $I$ and $S$ are the spin angular momentum operators, $I_{z}$ and $S_{z}$ are z-components of the spin angular momentum $I$ and $S$, respectively. We could also refer to this Hamiltonian as the "internal" Hamiltonian, since it depends only on the characteristics of the spin system. Once the internal Hamiltonian is known, by diagonalization of the Hamiltonian matrix it is possible to calculate the energy levels and determine the position in the spectrum of the resonance peaks. More details could be found in the literature; for instance in the books by Pople [102] and Corio [103], as well as in the review paper by Corio [109], many examples of spin systems $\left(\mathrm{AB}, \mathrm{A}_{2} \mathrm{~B}, \mathrm{~A}_{3} \mathrm{~B}, \mathrm{~A}_{3} \mathrm{~B}_{2}, \mathrm{ABC}, \mathrm{ABX} \ldots\right)$ are discussed in depth; the spin system eigenvalues and eigenfunctions, their resonance frequencies and relative intensities are tabulated in great detail.

In Figure 2, a schematic representation of the energy levels in a one-spin system and a twospin system, is shown. In the one-spin system we have two spin states: i) the state denoted as $|+\rangle$, where the spin is aligned with the field and ii) the state denoted as $|-\rangle$, with the spin aligned against the field; in this latter state, the spin has a higher energy than in $|+\rangle$. Thus, the one-spin system has two energy levels and one transition, which yields one resonance peak (Figure 2, left panel).

For a two-spin system we have four spin states, which correspond to 1) two spins aligned with the field $\mid++>$, 2) two spins aligned against the field $\mid-->$, and 3) \& 4) one spin aligned with and the other aligned against the field, $|-+>\&|+->$, respectively. Each state $(|++>|-,+>,|+->|-,->)$ is characterized by its energy level $\left(E_{1}, E_{2}, E_{3}, E_{4}\right.$, respectively) and its spin quantum number (1, 0, 0, and -1 , respectively). 
According to the selection rules of quantum mechanics (change in the spin system quantum number $= \pm 1$ ) there are the four allowed transitions, which are indicated by the arrows (Figure 2): $\mathrm{E}_{1} \rightarrow \mathrm{E}_{2}, \mathrm{E}_{1} \rightarrow \mathrm{E}_{3}, \mathrm{E}_{2} \rightarrow \mathrm{E}_{4}$ and $\mathrm{E}_{3} \rightarrow \mathrm{E}_{4}$.

In the case of two uncoupled spins (Figure 2, center panel), the transition $E_{1} \rightarrow E_{2}$, yields the same resonance peak as the transition $\mathrm{E}_{3} \rightarrow \mathrm{E}_{4}$; furthermore, the transition $\mathrm{E}_{1} \rightarrow \mathrm{E}_{3}$, yields the same resonance peak as the transition $\mathrm{E}_{2} \rightarrow \mathrm{E}_{4}$. The $J$ coupling modifies the value of the energy levels: the two singlets become now two doublets (Figure 2, right panel), with the effect of the $J$ coupling on the energy levels becoming more important when the spins are strongly coupled.

In addition to the four allowed transitions, there are two 'forbidden' transitions: $E_{2} \rightarrow E_{3}$, and $\mathrm{E}_{1} \rightarrow \mathrm{E}_{4}$, where the change in quantum number is zero and 2, respectively; for this reason, these transition are denoted as zero-quantum and double-quantum transitions.

With regard to the spin system Hamiltonian: in a previous section, we have discussed the difference between the weakly coupled and strongly coupled spin systems. Now we will discuss the difference between the Hamiltonian of the weakly coupled and the strongly coupled two-spin system.

In the strongly coupled system, the scalar interaction term $J I \cdot S$ is the scalar product of the two angular momentum operators, that is:

$$
J I \cdot S=J\left(I_{x} S_{x}+I_{y} S_{y}+I_{z} S_{z}\right)
$$

In the weakly coupled system, we can use the approximation that neglects the off-diagonal terms; in other words, the simplification of the scalar coupling yields:

$$
J I \cdot S \approx J\left(I_{z} S_{z}\right)
$$

Thus, the Hamiltonian of a weakly coupled two-spin system (an AX system) is written as:

$$
H=\omega_{A} I_{z}+\omega_{X} S_{Z}+J I_{z} \cdot S_{z}
$$

where $\omega_{A}$ and $\omega_{X}$ are the chemical shifts of the spin A and $X$, respectively, $J$ is the coupling constant, $I_{Z}$ and $S_{Z}$ are z-components of the spin angular momentum $I$ (spin A) and $S$ (spin $\mathrm{X})$, respectively. For sake of clarity we state again that we use the historical notation, where the angular momentum operators of a two-spin system (the A and X spins in this case) are denoted by the letter $I$ and $S$. The weakly coupled approximation greatly simplifies the analytical calculations, because now we deal with a diagonal matrix; the product operator formalism can be used in this case to obtain the MR signal. In the spin product basis, the representation of the Hamiltonian of a weakly and strongly coupled two-spin system is indicated here below, where the non-zero elements are denoted as "*": 


$$
H_{\text {weak }}=\left(\begin{array}{cccc}
* & 0 & 0 & 0 \\
0 & * & 0 & 0 \\
0 & 0 & * & 0 \\
0 & 0 & 0 & *
\end{array}\right) ; \quad H_{\text {strong }}=\left(\begin{array}{cccc}
* & 0 & 0 & 0 \\
0 & * & * & 0 \\
0 & * & * & 0 \\
0 & 0 & 0 & *
\end{array}\right) ;
$$

In contrast to the weakly coupled situation, the Hamiltonian of the strongly coupled two-spin system contains non-zero off-diagonal terms; these elements generate a coherence transfer occurring throughout the evolution periods. In other words, the strong coupling leads to coherence mixing, even in the absence of radiofrequency (RF) pulses.

It goes without saying that, when using the density matrix formalism, it is advantageous to write the $J$ coupling interaction always in its full form, that is:

$$
J_{A B} I \cdot S=J_{A B}\left(I_{x} S_{x}+I_{y} S_{y}+I_{z} S_{z}\right)
$$

and if the spin system is weakly coupled then automatically in the computational process the off-diagonal terms will have a negligible effect.

The second step is to investigate the response of the spin system to a given pulse sequence, such as the PRESS sequence, for instance. To achieve spatial localization of the signal, the PRESS sequence -as well as the STEAM sequence- employs three slice-selective RF pulses, applied in the presence of orthogonal gradients. The intersection of these three orthogonal slices yields the region of interest. The PRESS acquisition scheme can be written as follows:

$$
\text { PRESS sequence: } 90_{x}^{\circ}-t_{1}-180_{y}^{\circ}-t_{1}-t_{2}-180_{y}^{\circ}-t_{2}-\text { Acq }
$$

that is, a $90_{x}^{\circ}$ pulse is applied to the spin system, followed by a time interval $t_{1}$, then a pulse $180_{y}^{\circ}$ is applied and so on.

We have previously said that by diagonalizing the Hamiltonian we can obtain the energy levels and the eigenstates of the spin system; with regard to the eigenstates of the $J$ coupled two-spin system, what is the difference between the weakly coupled and the strongly coupled system? It can be shown that the eigenstates of the weakly coupled system are 1) $\mid++>, 2$ ) $\mid-->$, 3) $\mid+->$, and 4) $\mid-+>$. The eigenstates of the strongly coupled system are 1) $\mid++>, 2) \mid-->$, similarly to the weakly coupled case; the remaining two eigenstates are a linear combination of the states $\mid+->$ and $\mid-+>$, that is: 3) $a|+->+b|-+>$ and 4 ) $-b|+->+\mathrm{a}|-+>$, where $a$ and $\mathrm{b}$ are coefficients that depend on the chemical shifts and $J$ coupling.

Now we return to the density matrix simulations and we can highlight some of the steps of the overall procedure. From the classical physics viewpoint of the magnetic resonance phenomenon we know that, before applying the $90_{x}^{\circ}$ pulse, the equilibrium magnetization is along the z-direction. What is the quantum equivalent of the classical magnetization vector 
along $\mathrm{z}$ ? In the quantum mechanics framework, the $\mathrm{z}$-magnetization is represented by the $\mathrm{z}-$ component $\left(F_{z}=I_{z}+S_{z}\right)$ of the total spin angular momentum operator, $F$. Thus the density matrix before the $90_{x}^{\circ}$ pulse is equal to $F_{z}$ :

$$
\sigma(0)=F_{z}
$$

We then apply the $90_{x}^{\circ}$ pulse. At this stage, we have another form of energy interaction, i.e., the interaction between the RF pulse and the spin system; as a consequence, we do need to consider an additional term in the Hamiltonian; this term is active only during the duration of the RF pulse. During the RF pulse, the radiofrequency field is dominant over all other interactions, that is, the Zeeman and the $J$ coupling interactions. Thus, during the RF pulse we can neglect the Zeeman and the $J$ coupling terms and the total Hamiltonian consists only of the radiofrequency field energy. For sake of simplicity, the RF pulses are considered here as ideal rotation operators: the Hamiltonian corresponding to the $90_{x}^{\circ}$ pulse is then equal to $H=\frac{\pi}{2} F_{X}$. To calculate the density matrix, which we denote as $\sigma^{\prime}$, at the time $t$ just after the pulse, we use the formula given by the solution of the Liouville - von Neumann equation; that is, we multiply the initial density matrix to the left and to the right by the so-called time propagators $e^{-i t H}$ and $e^{i t H}$, respectively:

$$
\sigma^{\prime}=e^{-i \frac{\pi}{2} F_{x}} \sigma(0) e^{i \frac{\pi}{2} F_{x}}
$$

Since $\sigma(0)=F_{z}$, we can write:

$$
\sigma^{\prime}=e^{-i \frac{\pi}{2} F_{x}} F_{z} e^{i \frac{\pi}{2} F_{x}}
$$

and it can be shown that the matrix product above yields a density matrix $\sigma^{\prime}=F_{y}$. That is, similarly to the classical description of the MR experiment, where the $90_{x}^{\circ}$ pulse converts the $\mathrm{z}$-magnetization into the $\mathrm{y}$-magnetization, in the quantum world the $90_{x}^{\circ}$ pulse converts the quantum state represented by $F_{z}$ into $F_{y}$. So far, we have not gained much by applying the density matrix concept in comparison to the results that we could have obtained by the classical magnetization vector approach.

After the $90_{x}^{\circ}$ pulse, the evolution of the density matrix during the time interval $t_{1}$ is governed by the "internal" Hamiltonian, which consists of the Zeeman and the $J$ coupling interaction; here the classical picture of the magnetization vector breaks down. For the sake of completeness, it should be noted that there was one study where the authors included the $J$ coupling interaction into the Bloch equations, for an AB system [118]. These "Bloch-like equations" consisted of system of 15 first-order coupled differential equations; such approach, however, was quite cumbersome and furthemore was limited to the $\mathrm{AB}$ system. The $J$ 
coupling interaction is easily taken into account within the density matrix framework: we again apply the solution of the Liouville - von Neumann equation and the density matrix at the end of the time interval $t_{1}$, which we denote as $\sigma\left(t_{1}\right)$, is given by:

$$
\sigma\left(t_{1}\right)=e^{-i t_{1} H} \sigma^{\prime} e^{i t_{1} H}
$$

where $H$ is the internal Hamiltonian.

The same procedure is applied and repeated for all the other sequence blocks, that is, RF pulses and interpulse delays. To summarize, the density matrix at the end of the pulse sequence is:

$$
\sigma(t)=e^{-i t H} e^{-i t_{2} H} e^{-i \pi F_{y}} e^{-i t_{2} H} e^{-i t_{1} H} e^{-i \pi F_{y}} e^{-i t_{1} H} e^{-i \frac{\pi}{2} F_{x}} \sigma(0) e^{i \frac{\pi}{2} F_{x}} e^{i t_{1} H} e^{i \pi F_{y}} e^{i t_{1} H} e^{i t_{2} H} e^{i \pi F_{y}} e^{i t_{2} H} e^{i t H}
$$

or, in a more compact form:

$$
\sigma(t)=U^{-} \sigma(0) U^{+}
$$

with

$$
\begin{gathered}
U^{-}=e^{-i t H} e^{-i t_{2} H} e^{-i \pi F_{y}} e^{-i t_{2} H} e^{-i t_{1} H} e^{-i \pi F_{y}} e^{-i t_{1} H} e^{-i \frac{\pi}{2} F_{x}} \\
U^{+}=e^{i \frac{\pi}{2} F_{x}} e^{i t_{1} H} e^{i \pi F_{y}} e^{i t_{1} H} e^{i t_{2} H} e^{i \pi F_{y}} e^{i t_{2} H} e^{i t H}
\end{gathered}
$$

where $U$ is the time evolution operator (or propagator operator).

Overall, the trick at the core of the density matrix calculations and simulations consists into breaking up the pulse sequence in time intervals where the Hamiltonian is time independent so that we can apply the simple solution of the Liouville - von Neumann equation for timeindependent Hamiltonians. Simply put, the density matrix simulations boils down ultimately to a multiplication of matrices. This formalism can be easily extended to account for shaped RF pulses and magnetic field gradients.

We have now obtained the density matrix for a given pulse sequence (PRESS) and specific sequence parameters (i.e., the interpulse delays). The final step consists in calculating the physical quantity of interest, that is, the transverse magnetization. For instance, we can calculate the $\mathrm{x}$-component of the total magnetization, which is represented by the angular momentum operator $F_{x}$. To his aim, we apply the general equation to obtain the average value of a given physical quantity:

$$
<F_{x}>=\operatorname{Tr}\left(\sigma F_{x}\right)
$$

that is, the $\mathrm{x}$-component of the total magnetization is given by the trace of the product of the density matrix and angular momentum operator $F_{x}$.

Typically, for standard quadrature detection the real and imaginary components of the transverse magnetization are measured; therefore we are interested in measuring the 
transverse magnetization $F_{x}+i F_{y}$ (this novel operator is generally referred to as $F_{+}$). The average value of the transverse magnetization is then equal to:

$$
<F_{+}>=\operatorname{Tr}\left(\sigma F_{+}\right)
$$

By calculating $F_{x}+i F_{y}$ at multiple time points after the last $t_{2}$ delay, we sample the free induction decay. The Fourier transform of the free induction decay yields the MR spectrum and... that's that: we have performed the density matrix simulation for a strongly coupled two spin system, under PRESS excitation. We will show some example of simulations and experimental data for an $\mathrm{AB}$ spin system in the Experiments section.

We will now examine the case of metabolites characterized by a spin system other than the AB system. The first step consists in modifying accordingly the internal Hamiltonian. For instance, for a spin system with three strongly coupled spins (ABC spin system) the Hamiltonian is written as:

$$
H=\omega_{A} A_{z}+\omega_{B} B_{z}+\omega_{C} C_{z}+J_{A B} A \cdot B+J_{B C} B \cdot C+J_{A C} A \cdot C
$$

Where i) $\omega_{A}, \omega_{B}$ and $\omega_{C}$ are the chemical shifts of the spins $A, B$ and $C$, respectively, ii) $J_{A B}$, $J_{B C}, J_{A C}$ is the $J$ coupling constant between the spin $A \& B, B \& C$, and $A \& C$, respectively iii) $A, B$ and $C$ are the spin angular momentum operators of the spins $A, B$ and $C$, respectively. We abandon here the historical notation of $I$ and $S$ for the angular momentum of each spin, since we have more than two spins and it is more convenient to use a more intuitive notation. When one of the three spins is weakly coupled to the other two spins (ABX spin system) the Hamiltonian becomes:

$$
H=\omega_{A} A_{z}+\omega_{B} B_{z}+\omega_{X} X_{Z}+J_{A B} A \cdot B+J_{B X} B_{Z} \cdot X_{Z}+J_{A X} A_{Z} \cdot X_{Z}
$$

It should also be noted that when the number of spins changes, the dimensionality of the matrices changes too, since the number of quantum states is equal to $2^{n}$, where $n$ is the number of spins. For a single spin (with quantum number 1/2) the $\mathrm{x}, \mathrm{y}$, and $\mathrm{z}$ magnetization is represented by $2 \times 2$ matrices (the Pauli spin matrices) and there are two states. In the two-spin system there are four states and the dimension of the matrices is $4 \times 4$; for a three-spin system, we have eight states $(|+++>|+,+->, \ldots, \mid--->)$ and the dimension of the matrices is $8 \times 8$ and so on.

Once the internal Hamiltonian is defined, the procedure to perform the simulation is exactly the same as the one described for the AB spin system. Naturally, density matrix simulations allows also for investigations of metabolites with spin systems of higher complexity than the three-spin systems. Examples include the molecule of glutamate, glutamine, myo-inositol. For instance, myo-inositol consists of six $\mathrm{CH}$ groups that can be modeled as an $\mathrm{AM}_{2} \mathrm{~N}_{2} \mathrm{P}$ spin 
system: the $\mathrm{M}$ protons resonate at $3.52 \mathrm{ppm}\left(\mathrm{M}_{2}\right)$, the $\mathrm{N}$ at $3.61 \mathrm{ppm}\left(\mathrm{N}_{2}\right)$, while the $\mathrm{A}$ and $\mathrm{P}$ protons resonate at 4.05 and $3.27 \mathrm{ppm}$, respectively. The $J$ coupling constant between the $\mathrm{M}_{2}$ and $\mathrm{N}_{2}$ protons is equal to $9 \mathrm{~Hz}$ (the other $J$ coupling constants are given in Ref. 26), thus the $\mathrm{M}$ and $\mathrm{N}$ protons are strongly coupled even at the magnetic field strength of $9.4 \mathrm{~T}$.

In the section Quantum mechanics we have discussed the physical meaning of the elements of the density matrix. Now we can consider the specific case of a two-spin system. The basis consisting of the four product states $(|++>|+,->, \mid+->$ and $\mid-->)$ is the basis typically chosen to represent the density matrix of a two-spin system (AX or $A B$; in the notation " $\mid++>$ " the first symbol refers to the spin A and the second to the spin $\mathrm{X}$ ):

\begin{tabular}{|c|c|c|c|c|}
\hline$A X$ & $\mid++>$ & $\mid+->$ & $\mid-+>$ & $\mid-->$ \\
\hline$<++1$ & $\mathbf{P}_{1}$ & Single $\mathbf{Q}_{\mathbf{x}}$ & Single $\mathbf{Q}_{\mathbf{A}}$ & Double $\mathbf{Q}_{\mathrm{Ax}}$ \\
\hline$<+-1$ & Single $Q_{x}$ & $\mathbf{P}_{2}$ & Zero $\mathbf{Q}_{\mathbf{A X}}$ & Single $\mathbf{Q}_{\mathbf{A}}$ \\
\hline$<-+1$ & Single $\mathrm{Q}_{\mathrm{A}}$ & Zero QAX & $\mathbf{P}_{3}$ & Single $Q_{x}$ \\
\hline$<--1$ & Double $\mathrm{Q}_{\mathrm{AX}}$ & Single $\mathrm{Q}_{\mathrm{A}}$ & Single $Q_{x}$ & $\mathbf{P}_{4}$ \\
\hline
\end{tabular}

The matrix elements below the diagonal, which are the complex conjugate of the corresponding elements above the diagonal, are depicted in gray font. The diagonal elements represent the probability that the system is in that given state; in other words, $\mathbf{P}_{\mathbf{1}}, \mathbf{P}_{\mathbf{2}}, \mathbf{P}_{\mathbf{3}}$ and $\mathbf{P}_{\mathbf{4}}$ are the populations in the states $|++>|+,->, \mid+->$ and $\mid-->$, respectively. The off-diagonal elements, typically denoted as "coherences", connect two different quantum states: for instance, consider the element in the first row \& second column, the "Single $\mathrm{Qx}_{\mathrm{x}}$ " or single quantum coherence $\mathrm{Q}_{\mathrm{x}}$. This element connects the quantum state where the spin $\mathrm{A}$ and $\mathrm{X}$ are aligned with the field, $\mid++>$, and the state where the spin $\mathrm{A}$ is aligned with the field and $\mathrm{X}$ is aligned against the field, $\mid+->$; this corresponds to a quantum transition of the spin X. Similar argument can be applied to the "Single $\mathrm{Q}_{\mathrm{A}}$ " that connects two states which yield a transition for the spin $\mathrm{A}$. The "Zero $\mathrm{Q}_{\mathrm{AX}}$ " and "Double $\mathrm{Q}_{\mathrm{AX}}$ " are the matrix elements that connect two states where the difference in spin quantum number is equal to zero and 2 , respectively. 


\section{Experiments}

In this section we show some examples of density matrix simulations for an $\mathrm{AB}$ spin system; again, in the last decade the density matrix simulations have been extensively used in MRS, so the reader can find many other examples in the previously mentioned literature. We consider the $\mathrm{AB}$ spin system of citrate at $3 \mathrm{~T}$; the pulse sequence is a modified PRESS with the following RF pulses and interpulse delays:

$$
90_{x}^{\circ}-t_{1}-180_{y}^{\circ}-t_{1}-t_{2}-180_{y}^{\circ}-t_{2}-t_{3}-180_{y}^{\circ}-t_{3}-A c q
$$

It should be noted that the additional refocusing pulse, inserted into the standard PRESS sequence, helps minimizing the dephasing induced by the $J$ coupling so that a more favorable lineshape could be obtained at moderate echo times [119, 120].

The modified PRESS was implemented on a 3T whole body MR system (Siemens, Erlangen, Germany). Localized MR spectroscopy ( $2 \times 2 \times 2 \mathrm{~cm}^{3}$ volume of interest) was performed on a phantom containing citrate and creatine; the latter metabolite was added to the solution to provide a singlet signal for amplitude and phase reference. Analytical calculations were perfomed to derive the transverse magnetization of the $A B$ system for comparison with the experimental data and density matrix simulations [101]. Simulated free induction decays were generated for 1024 points over an acquisition time of $1 \mathrm{~s}$ and MR spectra were obtained by Fourier transform of the time signal. Figure 3 shows MR spectra obtained with the interpulse delays $\left[t_{1}, t_{2}, t_{3}\right]=[20,2,18] \mathrm{ms}$. It can be noted that the inner lines of citrate are in phase with the creatine signal. The result of the density matrix simulation is in excellent agreement with the analytical calculations and experimental data (Figure 3). With a different set of interpulse delays, $\left[t_{1}, t_{2}, t_{3}\right]=[20,18,2] \mathrm{ms}$, keeping the same total echo time of $80 \mathrm{~ms}$, a very different MR spectrum was obtained (Figure 4) with the inner lines of citrate being in opposite phase with respect to the creatine signal.

One observation is noteworthy when comparing the spectra of Figure 3 with those of Figure 4: the signal intensity of a strongly coupled spin system has a substantial dependence on the interpulse delays of PRESS sequences. This type of signal modulation, occurring at a constant echo time, is a unique characteristic of strongly coupled systems and could be exploited for editing purposes $[90,100]$. Within the context of the density matrix formalism, the source of this signal modulation is to be found in the non-zero off-diagonal terms of the strongly coupled Hamiltonian. These elements, which are absent in weakly coupled Hamiltonians, generate a coherence mixing during the interpulse delays. 
The density matrix simulations of MR spectra, as presented here, provide the basis for optimizing the detection of metabolites. The goal is to find the optimal sequence and sequence parameters that would yield a convenient lineshape for the metabolite of interest. Early investigations of metabolite optimization were limited to the assessment of the lineshape as a function of echo time only; it is well know that the signal intensity of weakly coupled systems is a function of the total echo time and is independent of the individual interpulse delays. However in strongly coupled systems, as we have just shown, it is necessary to simulate the MR spectra for different values of interpulse delays as well. When investigating all possible combinations of interpulse delays, the one-dimensional solution space $f(T E)$ becomes a twodimensional space $f\left(t_{1}, t_{2}\right)$ in the case of the standard PRESS sequence (and a threedimensional space $f\left(t_{1}, t_{2}, t_{3}\right)$ in the case of the modified PRESS sequence). As a consequence, the optimization procedure would easily result into the simulation of thousands of spectra. A convenient visual representation of all these data can be achieved with the following two steps: i) calculate the signal intensity (i.e., area under the resonance signal) of each spectrum obtained with a set of parameters $\left(\left[t_{1}, t_{2}\right]\right.$, in the standard PRESS sequence) and ii) represent the signal intensity as a function of $\left[t_{1}, t_{2}\right]$ in a $2 \mathrm{D}$ plot. How to proceed to accomplish the first step? The direct way is to simulate the free induction decay, perform the Fourier transform to obtain the MR spectrum and then perform the signal integration to determine the area under the peak. This approach, which requires the simulation of the MR spectra for all $\left[t_{1}, t_{2}\right]$, is rather impractical. A different approach consists in simulating just the first point of the free induction decay for all $\left[t_{1}, t_{2}\right]$; it can be easily shown (see Appendix 2) that the area under the resonance signal is equal to the value of the first point of the free induction decay. This latter method substantially decreases the computational time of the density matrix simulations.

Here we show density matrix simulations (Figure 5) of the citrate signal for the standard PRESS:

$$
90_{x}^{\circ}-t_{1}-180_{y}^{\circ}-t_{1}-t_{2}-180_{y}^{\circ}-t_{2}-A c q
$$

at the field strength of $3 \mathrm{~T}$. The signal intensity is plotted as a function of $\mathrm{TE}_{1}$ and $\mathrm{TE}_{2}$, where $\mathrm{TE}_{1}=2 t_{1}$ and $\mathrm{TE}_{2}=2 t_{2}$. It should be noted that the signal intensity of $90000 \mathrm{MR}$ spectra is now displayed in a compact way, in Figure 5. The results of the density matrix simulations shown in Figure 5 allow us to identify the interpulse delays that maximize the signal intensity of citrate; in other words, these results allow us to optimize citrate detection. 
The same simulation was performed at the magnetic field of $11.7 \mathrm{~T}$ (Figure 6). As in Figure 5, two representations of the same data are displayed. The $\mathrm{TE}_{1}$ is on the $\mathrm{x}$-axis and the $\mathrm{TE}_{2}$ is on the y-axis. For a given TE, the points of the flat surface graph with same TE values are on the diagonal line described by the equation $y=-x+T E$ (that is: $T E_{2}=-T E_{1}+T E$ ). In the case of a weakly coupled spin system, there would be no signal oscillation along these diagonal lines, that is, the "waves" would be smooth with no bumps on the crests and valleys. The signal oscillations at constant TE, to be ascribed to the coherence mixing induced by the non-diagonal elements of the Hamiltonian, show that the effects of the strong coupling in the spin system of citrate are still present at a very high magnetic field. 


\section{Appendix 1}

Here we report the matrix representation of the angular momentum operators $I_{x}, I_{y}$ and $I_{z}$, for spins with quantum number $1 / 2$.

$I_{x}, I_{y}$ and $I_{z}$ are the quantum mechanics analogues of the $x-, y-$, and z-component, respectively, of the (classical physics) magnetization vector. These operators are the building blocks that allow for the assessment of the signal intensity by density matrix simulations.

Let's introduce the Pauli spin matrices:

$g_{x}=\frac{1}{2}\left(\begin{array}{ll}0 & 1 \\ 1 & 0\end{array}\right) \quad g_{y}=\frac{i}{2}\left(\begin{array}{rr}0 & -1 \\ 1 & 0\end{array}\right) \quad g_{z}=\frac{1}{2}\left(\begin{array}{rr}1 & 0 \\ 0 & -1\end{array}\right)$

It should be noted that the coefficient of the $g_{y}$ matrix is $i / 2$, where $i$ is the imaginary unit; on the other hand, for the $g_{x}$ and $g_{z}$ matrices the coefficient is $1 / 2$.

For a single spin (spin I), the angular momentum operators $I_{x}, I_{y}$ and $I_{z}$ are represented by the Pauli spin matrices $\mathrm{I}_{\mathrm{x}}{ }^{*}, \mathrm{I}_{\mathrm{y}}{ }^{*}$ and $\mathrm{I}_{\mathrm{z}}{ }^{*}$, respectively:

$$
\begin{aligned}
& I_{x}=g_{x} \\
& I_{y}=g_{y} \\
& I_{z}=g_{z}
\end{aligned}
$$

Thus, the angular momentum operators for a single spin consist of $2 \times 2$ matrices.

In the case of a two-spin system (spin I and spin S), the matrix representation of the angular momentum operators needs to be modified when compared to the case of a single spin. We start with the spin I. The representation of the angular momentum operator for the spin I is obtained by the Kronecker product of Pauli matrices with the identity matrix E. We should point out that the Kronecker product of two matrices of dimensions $2 \times 2$ generates a $4 \times 4$ matrix.

The angular momentum operators $\mathrm{I}_{\mathrm{x}}$ is given by:

$$
I_{x}=g_{x} \otimes E
$$

where the symbol $\otimes$ indicates the Kronecker product. This formula can be developed as follows: 


$$
E=\left(\begin{array}{ll}
1 & 0 \\
0 & 1
\end{array}\right)
$$

$I_{x}=g_{x} \otimes E=\frac{1}{2}\left(\begin{array}{ll}0 & 1 \\ 1 & 0\end{array}\right) \otimes E=\frac{1}{2}\left(\begin{array}{ll}0 \cdot E & 1 \cdot E \\ 1 \cdot E & 0 \cdot E\end{array}\right)=\frac{1}{2}\left(\begin{array}{llll}0 \cdot 1 & 0 \cdot 0 & 1 \cdot 1 & 1 \cdot 0 \\ 0 \cdot 0 & 0 \cdot 1 & 1 \cdot 0 & 1 \cdot 1 \\ 1 \cdot 1 & 1 \cdot 0 & 0 \cdot 1 & 0 \cdot 0 \\ 1 \cdot 0 & 1 \cdot 1 & 0 \cdot 0 & 0 \cdot 1\end{array}\right) \Rightarrow$ $I_{x}=\frac{1}{2}\left(\begin{array}{llll}0 & 0 & 1 & 0 \\ 0 & 0 & 0 & 1 \\ 1 & 0 & 0 & 0 \\ 0 & 1 & 0 & 0\end{array}\right)$

Thus, the representation of the $\mathrm{x}$-component of angular momentum operator corresponding to the spin I, for a two-spin system, is given by $4 \times 4$ matrix:

The angular momentum operators of spin $S$ are obtained by inverting the order of the elements of the Kronecker product, that is, by taking the product of the identity matrix E with Pauli matrix:

$$
S_{x}=E \otimes g_{x}
$$

Thus, the angular momentum operator $S_{X}$ is:

$S_{x}=E \otimes g_{x}=\left(\begin{array}{ll}1 & 0 \\ 0 & 1\end{array}\right) \otimes g_{x}=\left(\begin{array}{ll}1 \cdot g_{x} & 0 \cdot g_{x} \\ 0 \cdot g_{x} & 1 \cdot g_{x}\end{array}\right)=\frac{1}{2}\left(\begin{array}{llll}1 \cdot 0 & 1 \cdot 1 & 0 \cdot 0 & 0 \cdot 1 \\ 1 \cdot 1 & 1 \cdot 0 & 0 \cdot 1 & 0 \cdot 0 \\ 0 \cdot 0 & 0 \cdot 1 & 1 \cdot 0 & 1 \cdot 1 \\ 0 \cdot 1 & 0 \cdot 0 & 1 \cdot 1 & 1 \cdot 0\end{array}\right) \Rightarrow$ $S_{x}=\frac{1}{2}\left(\begin{array}{llll}0 & 1 & 0 & 0 \\ 1 & 0 & 0 & 0 \\ 0 & 0 & 0 & 1 \\ 0 & 0 & 1 & 0\end{array}\right)$

Here we report the matrix representation of the angular momentum operators $I_{x}, I_{y}$ and $I_{z}$, for spins with quantum number $1 / 2$.

The matrix representation of the $y$ - and z-component of the angular momentum operators for the spin I and $\mathrm{S}$ can be obtained in the same manner, that is:

$$
\begin{aligned}
& I_{y}=g_{y} \otimes E \\
& S_{y}=E \otimes g_{y}
\end{aligned}
$$


and

$$
\begin{aligned}
& I_{z}=g_{z} \otimes E \\
& S_{z}=E \otimes g_{z}
\end{aligned}
$$

To summarize, in the case of a two-spin system (spin I and spin S), the matrix representation of the angular momentum operators $\mathrm{I}_{\mathrm{x}}, \mathrm{I}_{\mathrm{y}}$ and $\mathrm{I}_{\mathrm{z}}$ and $\mathrm{S}_{\mathrm{x}}, \mathrm{S}_{\mathrm{y}}$ and $\mathrm{S}_{\mathrm{z}}$ is:

$$
\begin{aligned}
I_{x}=\frac{1}{2}\left(\begin{array}{llll}
0 & 0 & 1 & 0 \\
0 & 0 & 0 & 1 \\
1 & 0 & 0 & 0 \\
0 & 1 & 0 & 0
\end{array}\right) & I_{y}=\frac{i}{2}\left(\begin{array}{rrrr}
0 & 0 & -1 & 0 \\
0 & 0 & 0 & -1 \\
1 & 0 & 0 & 0 \\
0 & 1 & 0 & 0
\end{array}\right) \\
S_{x}=\frac{1}{2}\left(\begin{array}{llll}
0 & 1 & 0 & 0 \\
1 & 0 & 0 & 0 \\
0 & 0 & 0 & 1 \\
0 & 0 & 1 & 0
\end{array}\right) & I_{z}=\frac{1}{2}\left(\begin{array}{ccccc}
1 & 0 & 0 & 0 \\
0 & 1 & 0 & 0 \\
1 & 0 & -1 & 0 \\
0 & 1 & 0 & -1
\end{array}\right) \\
&
\end{aligned}
$$

The total spin angular momentum operator is then given by $F_{x}=I_{x}+S_{x}, F_{y}=I_{y}+S_{y}$ and $F_{z}=I_{z}+S_{z}:$

$$
F_{x}=\frac{1}{2}\left(\begin{array}{llll}
0 & 1 & 1 & 0 \\
1 & 0 & 0 & 1 \\
1 & 0 & 0 & 1 \\
0 & 1 & 1 & 0
\end{array}\right) \quad F_{y}=\frac{i}{2}\left(\begin{array}{rrrr}
0 & -1 & -1 & 0 \\
1 & 0 & 0 & -1 \\
1 & 0 & 0 & -1 \\
0 & 1 & 1 & 0
\end{array}\right) \quad F_{z}=\left(\begin{array}{rrrr}
1 & 0 & 0 & 0 \\
0 & 0 & 0 & 0 \\
0 & 0 & 0 & 0 \\
0 & 0 & 0 & -1
\end{array}\right)
$$




\section{Appendix 2}

Here we provide the derivation of a useful formula to obtain the signal intensity, i.e., the area under the curve of the frequency-domain signal. The area under the resonance curve is by definition the integral of the frequency-domain signal $F(\omega)$ :

$$
A=\int_{\omega} F(\omega) d \omega
$$

Using the definition of Fourier transform $(f(t)$ is the time-domain signal):

$$
F(\omega)=\int_{t} f(t) e^{-i \omega t} d t
$$

and exchanging the time and frequency integrals in the formula below we obtain:

$$
A=\int_{\omega} \int_{t} f(t) e^{-i \omega t} d t d \omega=\int_{t} \int_{\omega} f(t) e^{-i \omega t} d \omega d t=\int_{t} f(t)\left[\int_{\omega} e^{-i \omega t} d \omega\right] d t
$$

The integral in the square brackets is the Dirac delta function, $\delta(t)$; inserting $\delta(t)$ in the equation above and using the following property of the Dirac delta function:

$$
\int_{t} f(t) \delta(t) d t=f(0)
$$

the formula becomes:

$$
A=\int_{t} f(t) \delta(t) d t=f(0)
$$

that is, the area under the signal in the frequency domain is equal to the value of the first point of the time-domain signal. 


\section{Acknowledgments}

My first encounter with the density matrix formalism applied to magnetic resonance spectroscopy was during my $\mathrm{PhD}$ thesis work. Many years have passed by and I am still indebted to my thesis director who introduced me to the wonders of the density matrix -or, as he often referred to it as, The Destiny Matrix! 


\section{References}

[1] Smith, W. (1884). A Dictionary of Greek and Roman biography and mythology. Harper \& Brothers, New York, USA.

[2] Purcell, E. M., Torrey, H. C., \& Pound, R. V. (1946). Resonance absorption by nuclear magnetic moments in a solid. Physical review, 69(1-2), 37.

[3] Bloch, F. (1946). Nuclear induction. Physical review, 70(7-8), 460-474.

[4] Bloch F., Hansen W. W., \& Packard M. (1946). The nuclear induction experiment. Physical review, 70(7-8), 474-480.

[5] Rigden, J. S. (1986). Quantum states and precession: The two discoveries of NMR. Reviews of modern physics, 58(2), 433-438.

[6] Pound, R. V. (1999). From radar to nuclear magnetic resonance. Reviews of modern physics, 71(2), S54.

[7] Ramsey, N. F. (1999). Early history of magnetic resonance. Physics in perspective, $1(2), 123-135$.

[8] Bloch, F. (1951). Nuclear induction. Physica, 17(3-4), 272-281.

[9] Gorter, C. J. (1936). Negative result of an attempt to detect nuclear magnetic spins. Physica, 3(9), 995-998.

[10] Gorter, C. J., \& Broer, L. J. F. (1942). Negative result of an attempt to observe nuclear magnetic resonance in solids. Physica, 9(6), 591-596.

[11] Van Vleck (ed.). International Symposium on Electron and Nuclear Magnetic Resonance (1969, Melbourne). Plenum Press, New York, 1970.

[12] Bottomley, P. A. (1989). Human in vivo NMR spectroscopy in diagnostic medicine: clinical tool or research probe?. Radiology, 170(1), 1-15.

[13] Smith, I. C., \& Stewart, L. C. (2002). Magnetic resonance spectroscopy in medicine: clinical impact. Progress in nuclear magnetic resonance spectroscopy, 40(1), 1-34.

[14] Barker, P. B., \& Lin, D. D. (2006). In vivo proton MR spectroscopy of the human brain. Progress in nuclear magnetic resonance spectroscopy, 49(2), 99-128.

[15] Kreis, R. (1997). Quantitative localized 1H MR spectroscopy for clinical use. Progress in nuclear magnetic resonance spectroscopy, 31(2), 155-195.

[16] Kwock, L., Smith, J. K., Castillo, M., Ewend, M. G., Collichio, F., Morris, D. E., ... \& Cush, S. (2006). Clinical role of proton magnetic resonance spectroscopy in oncology: brain, breast, and prostate cancer. The lancet oncology, 7(10), 859-868. 
[17] Rothman, D. L., Petroff, O. A., Behar, K. L., \& Mattson, R. H. (1993). Localized 1H NMR measurements of gamma-aminobutyric acid in human brain in vivo. Proceedings of the national academy of sciences, 90(12), 5662-5666.

[18] Petroff, O. A., Behar, K. L., Mattson, R. H., \& Rothman, D. L. (1996). Human Brain $\gamma$-Aminobutyric Acid Levels and Seizure Control Following Initiation of Vigabatrin Therapy. Journal of neurochemistry, 67(6), 2399-2404.

[19] Shen, J., Yang, J., Choi, I. Y., Li, S. S., \& Chen, Z. (2004). A new strategy for in vivo spectral editing. Application to GABA editing using selective homonuclear polarization transfer spectroscopy. Journal of magnetic resonance, 170(2), 290-298.

[20] Bielicki, G., Chassain, C., Renou, J. P., Farges, M. C., Vasson, M. P., Eschalier, A., \& Durif, F. (2004). Brain GABA editing by localized in vivo $1 \mathrm{H}$ magnetic resonance spectroscopy. NMR in biomedicine, 17(2), 60-68.

[21] Near, J., Evans, C. J., Puts, N. A., Barker, P. B., \& Edden, R. A. (2013). J-difference editing of gamma-aminobutyric acid (GABA): Simulated and experimental multiplet patterns. Magnetic resonance in medicine, 70(5), 1183-1191.

[22] Prichard, J., Rothman, D., Novotny, E., Petroff, O., Kuwabara, T., Avison, M., ... \& Shulman, R. (1991). Lactate rise detected by $1 \mathrm{H}$ NMR in human visual cortex during physiologic stimulation. Proceedings of the national academy of sciences, 88(13), 5829-5831.

[23] Frahm, J., Krüger, G., Merboldt, K. D., \& Kleinschmidt, A. (1996). Dynamic uncoupling and recoupling of perfusion and oxidative metabolism during focal brain activation in man. Magnetic resonance in medicine, 35(2), 143-148.

[24] Lin, Y., Stephenson, M. C., Xin, L., Napolitano, A., \& Morris, P. G. (2012). Investigating the metabolic changes due to visual stimulation using functional proton magnetic resonance spectroscopy at 7 T. Journal of cerebral blood flow \& metabolism, 32(8), 1484-1495.

[25] Cai, K., Nanga, R. P., Lamprou, L., Schinstine, C., Elliott, M., Hariharan, H., ... \& Epperson, C. N. (2012). The impact of gabapentin administration on brain GABA and glutamate concentrations: a 7T 1H-MRS study. Neuropsychopharmacology, 37(13), 27642771.

[26] Govindaraju, V., Young, K., \& Maudsley, A. A. (2000). Proton NMR chemical shifts and coupling constants for brain metabolites. NMR in biomedicine, 13(3), 129-153.

[27] Kurhanewicz, J., Vigneron, D. B., Nelson, S. J., Hricak, H., MacDonald, J. M., Konety, B., \& Narayan, P. (1995). Citrate as an in vivo marker to discriminate prostate cancer 
from benign prostatic hyperplasia and normal prostate peripheral zone: detection via localized proton spectroscopy. Urology, 45(3), 459-466.

[28] van der Graaf, M., van den Boogert, H. J., Jager, G. J., Barentsz, J. O., \& Heerschap, A. (1999). Human Prostate: Multisection Proton MR Spectroscopic Imaging with a Single Spin-Echo Sequence-Preliminary Experience 1. Radiology, 213(3), 919-925.

[29] Mulkern, R. V., \& Bowers, J. L. (1993). Calculating spectral modulations of AB systems during PRESS acquisitions. Magnetic resonance in medicine, 30(4), 518-519.

[30] Mulkern, R. V., Bowers, J. L., Peled, S., \& Williamson, D. S. (1996). Density-matrix calculations of the $1.5 \mathrm{~T}$ citrate signal acquired with volume-localized STEAM sequences. Journal of magnetic resonance, Series B, 110(3), 255-266.

[31] Schick, F., Eismann, B., Jung, W. I., Bongers, H., Bunse, M., \& Lutz, O. (1993). Comparison of localized proton NMR signals of skeletal muscle and fat tissue in vivo: two lipid compartments in muscle tissue. Magnetic resonance in medicine, 29(2), 158-167.

[32] Boesch, C., Slotboom, J., Hoppeler, H., \& Kreis, R. (1997). In vivo determination of intra-myocellular lipids in human muscle by means of localized 1H-MR-spectroscopy. Magnetic resonance in medicine, 37(4), 484-493.

[33] Szczepaniak, L. S., Babcock, E. E., Schick, F., Dobbins, R. L., Garg, A., Burns, D. K., ... \& Stein, D. T. (1999). Measurement of intracellular triglyceride stores by H spectroscopy: validation in vivo. American journal of physiology-endocrinology and metabolism, 276(5), E977-E989.

[34] Hamilton, G., Yokoo, T., Bydder, M., Cruite, I., Schroeder, M. E., Sirlin, C. B., \& Middleton, M. S. (2011). In vivo characterization of the liver fat 1H MR spectrum. NMR in biomedicine, 24(7), 784-790.

[35] Roebuck, J. R., Cecil, K. M., Schnall, M. D., \& Lenkinski, R. E. (1998). Human breast lesions: characterization with proton MR spectroscopy. Radiology, 209(1), 269-275.

[36] Kvistad, K. A., Bakken, I. J., Gribbestad, I. S., Ehrnholm, B., Lundgren, S., Fjøsne, H. E., \& Haraldseth, O. (1999). Characterization of neoplastic and normal human breast tissues with in vivo 1H MR spectroscopy. Journal of magnetic resonance imaging, 10(2), 159-164.

[37] Bolan, P. J., Meisamy, S., Baker, E. H., Lin, J., Emory, T., Nelson, M., ... \& Garwood, M. (2003). In vivo quantification of choline compounds in the breast with $1 \mathrm{H}$ MR spectroscopy. Magnetic resonance in medicine, 50(6), 1134-1143. 
[38] Bougnoux, P., Hajjaji, N., Maheo, K., Couet, C., \& Chevalier, S. (2010). Fatty acids and breast cancer: sensitization to treatments and prevention of metastatic re-growth. Progress in lipid research, 49(1), 76-86.

[39] Dimitrov, I. E., Douglas, D., Ren, J., Smith, N. B., Webb, A. G., Sherry, A. D., \& Malloy, C. R. (2012). In vivo determination of human breast fat composition by $1 \mathrm{H}$ magnetic resonance spectroscopy at $7 \mathrm{~T}$. Magnetic resonance in medicine, 67(1), 20-26.

[40] Graaf, R. A., Klomp, D. W., Luijten, P. R., \& Boer, V. O. (2014). Intramolecular zeroquantum-coherence 2D NMR spectroscopy of lipids in the human breast at $7 \mathrm{~T}$. Magnetic resonance in medicine, 71(2), 451-457.

[41] Coum, A., Ouldamer, L., Noury, F., Barantin, L., Saint-Hilaire, A., Vilde, A., ... \& Gambarota, G. (2016). In vivo MR spectroscopy of human breast tissue: quantification of fatty acid composition at a clinical field strength $(3 \mathrm{~T})$. Magnetic resonance materials in physics, biology and medicine, 29(1):1-4.

[42] Hardy, P. A., Henkelman, R. M., Bishop, J. E., Poon, E. C. S., \& Plewes, D. B. (1992). Why fat is bright in rare and fast spin-echo imaging. Journal of magnetic resonance imaging, 2(5), 533-540.

[43] Stables, L. A., Kennan, R. P., Anderson, A. W., \& Gore, J. C. (1999). Density matrix simulations of the effects of $\mathbf{J}$ coupling in spin echo and fast spin echo imaging. Journal of magnetic resonance, 140(2), 305-314.

[44] Mulkern, R. V., Packard, A. B., \& Gambarota, G. (2003). Field dependence of the bright fat effect in fast spin echo imaging: theory and experiment. Proceedings of the international society for magnetic resonance in medicine (ISMRM), Vol. 11, p. 1107.

[45] Stokes, A. M., Feng, Y., Mitropoulos, T., \& Warren, W. S. (2013). Enhanced refocusing of fat signals using optimized multipulse echo sequences. Magnetic resonance in medicine, 69(4), 1044-1055.

[46] Cohen-Tannoudji, C., Diu, B., Laloë F. (1977). Quantum Mechanics. Wiley, NewYork.

[47] Gearhart, C. A. (2002). Planck, the Quantum, and the Historians. Physics in perspective, 4(2), 170-215.

[48] Hermann A. (1971). The Genesis of Quantum Theory : 1899 -1913. MIT Press, Cambridge, MA.

[49] Langevin P., de Broglie M. (1912). La théorie du rayonnement et les quanta, rapports et discussions, Gauthier-Villars, Paris. 
[50] Bacciagaluppi, G., \& Valentini, A. (2009). Quantum theory at the crossroads: reconsidering the 1927 Solvay conference. Cambridge University Press.

[51] Ter Haar, D. (1961). Theory and applications of the density matrix. Reports on progress in physics, 24(1), 304-362.

[52] Fano, U. (1957). Description of states in quantum mechanics by density matrix and operator techniques. Reviews of modern physics, 29(1), 74.

[53] van der Graaf, M., \& Heerschap, A. (1996). Effect of cation binding on the proton chemical shifts and the spin-spin coupling constant of citrate. Journal of magnetic resonance, Series B, 112(1), 58-62.

[54] Gambarota, G., Bondon, A., Le Floch, M., Mulkern, R. V., \& Saint-Jalmes, H. (2013). Selective spectral modulation of strongly coupled spins with an echo top refocusing pulse in PRESS sequences. Journal of magnetic resonance, 228, 76-80.

[55] Van de Ven, F. J. M., \& Hilbers, C. W. (1983). A simple formalism for the description of multiple-pulse experiments. Application to a weakly coupled two-spin ( $I=1 / 2)$ system. Journal of magnetic resonance (1969), 54(3), 512-520.

[56] Sørensen, O. W., Eich, G. W., Levitt, M. H., Bodenhausen, G., \& Ernst, R. R. (1984). Product operator formalism for the description of NMR pulse experiments. Progress in nuclear magnetic resonance spectroscopy, 16, 163-192.

[57] Frahm, J., Merboldt, K. D., \& Hänicke, W. (1987). Localized proton spectroscopy using stimulated echoes. Journal of magnetic resonance (1969), 72(3), 502-508.

[58] Bottomley, P. A. (1987). Spatial localization in NMR spectroscopy in vivo. Annals of the New York academy of sciences, 508(1), 333-348.

[59] Ernst, T. H., \& Hennig, J. (1991). Coupling effects in volume selective 1H spectroscopy of major brain metabolites. Magnetic resonance in medicine, 21(1), 82-96.

[60] Wilman, A. H., \& Allen, P. S. (1995). The response of the strongly coupled AB system of citrate to typical $1 \mathrm{H}$ MRS localization sequences. Journal of magnetic resonance, Series B, 107(1), 25-33.

[61] Thompson, R. B., \& Allen, P. S. (1999). Sources of variability in the response of coupled spins to the PRESS sequence and their potential impact on metabolite quantification. Magnetic resonance in medicine, 41(6), 1162-1169.

[62] Thompson, R. B., \& Allen, P. S. (2001). Response of metabolites with coupled spins to the STEAM sequence. Magnetic resonance in medicine, 45(6), 955-965. 
[63] Yang, S., Hu, J., Kou, Z., \& Yang, Y. (2008). Spectral simplification for resolved glutamate and glutamine measurement using a standard STEAM sequence with optimized timing parameters at 3, 4, 4.7, 7, and 9.4 T. Magnetic resonance in medicine, 59(2), 236-244. [64] Maudsley, A. A., Govindaraju, V., Young, K., Aygula, Z. K., Pattany, P. M., Soher, B. J., \& Matson, G. B. (2005). Numerical simulation of PRESS localized MR spectroscopy. Journal of magnetic resonance, 173(1), 54-63.

[65] Kim, H., Thompson, R. B., Hanstock, C. C., \& Allen, P. S. (2005). Variability of metabolite yield using STEAM or PRESS sequences in vivo at $3.0 \mathrm{~T}$, illustrated with myoinositol. Magnetic resonance in medicine, 53(4), 760-769.

[66] Dou, W., Kaufmann, J., Li, M., Zhong, K., Walter, M., \& Speck, O. (2015). The separation of Gln and Glu in STEAM: a comparison study using short and long TEs/TMs at 3 and 7 T. Magnetic Resonance Materials in Physics, Biology and Medicine, 28(4), 395-405.

[67] Trabesinger, A. H., Meier, D., Dydak, U., Lamerichs, R., \& Boesiger, P. (2005). Optimizing PRESS localized citrate detection at 3 Tesla. Magnetic resonance in medicine, 54(1), 51-58.

[68] Banerjee, A., Ganji, S., Hulsey, K., Dimitrov, I., Maher, E., Ghose, S., ... \& Choi, C. (2012). Measurement of glycine in gray and white matter in the human brain in vivo by $1 \mathrm{H}$ MRS at 7.0 T. Magnetic resonance in medicine, 68(2), 325-331.

[69] Hurd, R., Sailasuta, N., Srinivasan, R., Vigneron, D. B., Pelletier, D., \& Nelson, S. J. (2004). Measurement of brain glutamate using TE-averaged PRESS at 3T. Magnetic resonance in medicine, 51(3), 435-440.

[70] Steinberg, J. D., \& Velan, S. S. (2013). Measuring glucose concentrations in the rat brain using echo-time-averaged point resolved spectroscopy at 7 tesla. Magnetic resonance in medicine, 70(2), 301-308.

[71] Scheenen, T. W., Gambarota, G., Weiland, E., Klomp, D. W., Fütterer, J. J., Barentsz, J. O., \& Heerschap, A. (2005). Optimal timing for in vivo 1H MR spectroscopic imaging of the human prostate at 3T. Magnetic resonance in medicine, 53(6), 1268-1274.

[72] Snyder, J., \& Wilman, A. (2010). Field strength dependence of PRESS timings for simultaneous detection of glutamate and glutamine from 1.5 to $7 \mathrm{~T}$. Journal of magnetic resonance, 203(1), 66-72.

[73] Yahya, A., Mädler, B., \& Fallone, B. G. (2008). Exploiting the chemical shift displacement effect in the detection of glutamate and glutamine (Glx) with PRESS. Journal of magnetic resonance, 191(1), 120-127. 
[74] Mullins, P. G., Chen, H., Xu, J., Caprihan, A., \& Gasparovic, C. (2008). Comparative reliability of proton spectroscopy techniques designed to improve detection of J-coupled metabolites. Magnetic resonance in medicine, 60(4), 964-969.

[75] Snyder, J., Hanstock, C. C., \& Wilman, A. H. (2009). Spectral editing of weakly coupled spins using variable flip angles in PRESS constant echo time difference spectroscopy: Application to GABA. Journal of magnetic resonance, 200(2), 245-250.

[76] Choi, C., Dimitrov, I. E., Douglas, D., Patel, A., Kaiser, L. G., Amezcua, C. A., \& Maher, E. A. (2010). Improvement of resolution for brain coupled metabolites by optimized 1H MRS at 7 T. NMR in biomedicine, 23(9), 1044-1052.

[77] Lally, N., An, L., Banerjee, D., Niciu, M. J., Luckenbaugh, D. A., Richards, E. M., ... \& Nugent, A. C. (2016). Reliability of 7T 1H-MRS measured human prefrontal cortex glutamate, glutamine, and glutathione signals using an adapted echo time optimized PRESS sequence: A between-and within-sessions investigation. Journal of magnetic resonance imaging, 43(1), 88-98.

[78] Toncelli, A., Noeske, R., Cosottini, M., Costagli, M., Domenici, V., Tiberi, G., \& Tosetti, M. (2015). STEAM-MiTiS: An MR spectroscopy method for the detection of scalarcoupled metabolites and its application to glutamate at $7 \mathrm{~T}$. Magnetic resonance in medicine, 74(6), 1515-1522.

[79] Napolitano, A., Kockenberger, W., \& Auer, D. P. (2013). Reliable gamma aminobutyric acid measurement using optimized PRESS at $3 \mathrm{~T}$. Magnetic resonance in medicine, 69(6), 1528-1533.

[80] Kaiser, L. G., Young, K., \& Matson, G. B. (2008). Numerical simulations of localized high field 1 H MR spectroscopy. Journal of magnetic resonance, 195(1), 67-75.

[81] Hu, J., Yang, S., Xuan, Y., Jiang, Q., Yang, Y., \& Haacke, E. M. (2007). Simultaneous detection of resolved glutamate, glutamine, and $\gamma$-aminobutyric acid at $4 \mathrm{~T}$. Journal of magnetic resonance, 185(2), 204-213.

[82] Hanstock, C. C., Coupland, N. J., \& Allen, P. S. (2002). GABA X2 multiplet measured pre-and post-administration of vigabatrin in human brain. Magnetic resonance in medicine, 48(4), 617-623.

[83] Kim, H., Wild, J. M., \& Allen, P. S. (2004). Strategy for the spectral filtering of myoinositol and other strongly coupled spins. Magnetic resonance in medicine, 51(2), 263-272. 
[84] Mayer, D., \& Spielman, D. M. (2005). Detection of glutamate in the human brain at 3 $\mathrm{T}$ using optimized constant time point resolved spectroscopy. Magnetic resonance in medicine, 54(2), 439-442.

[85] Kaiser, L. G., Young, K., Meyerhoff, D. J., Mueller, S. G., \& Matson, G. B. (2008). A detailed analysis of localized J-difference GABA editing: theoretical and experimental study at $4 \mathrm{~T}$. NMR in biomedicine, 21(1), 22-32.

[86] Kim, H., Thompson, R. B., \& Allen, P. S. (2012). Enhancement of spectral editing efficacy of multiple quantum filters in in vivo proton magnetic resonance spectroscopy. Journal of magnetic resonance, 223, 90-97.

[87] Trabesinger, A. H., Mueller, D. C., \& Boesiger, P. (2000). Single-quantum coherence filter for strongly coupled spin systems for localized $1 \mathrm{H}$ NMR spectroscopy. Journal of magnetic resonance, 145(2), 237-245.

[88] Pan, J. W., Avdievich, N., \& Hetherington, H. P. (2010). J-refocused coherence transfer spectroscopic imaging at $7 \mathrm{~T}$ in human brain. Magnetic resonance in medicine, 64(5), 1237-1246.

[89] Choi, I. Y., Lee, S. P., \& Shen, J. (2005). Selective homonuclear Hartmann-Hahn transfer method for in vivo spectral editing in the human brain. Magnetic resonance in medicine, 53(3), 503-510.

[90] Snyder, J., Thompson, R. B., \& Wilman, A. H. (2010). Difference spectroscopy using PRESS asymmetry: application to glutamate, glutamine, and myo-inositol. NMR in biomedicine, 23(1), 41-47.

[91] Yahya, A. (2009). Metabolite detection by proton magnetic resonance spectroscopy using PRESS. Progress in nuclear magnetic resonance spectroscopy, 55(3), 183-198.

[92] Puts, N. A., \& Edden, R. A. (2012). In vivo magnetic resonance spectroscopy of GABA: a methodological review. Progress in nuclear magnetic resonance spectroscopy, 60, 29-41.

[93] Ramadan, S., Lin, A., \& Stanwell, P. (2013). Glutamate and glutamine: a review of in vivo MRS in the human brain. NMR in biomedicine, 26(12), 1630-1646.

[94] Hattingen, E., Lanfermann, H., Quick, J., Franz, K., Zanella, F. E., \& Pilatus, U. (2009). 1H MR spectroscopic imaging with short and long echo time to discriminate glycine in glial tumours. Magnetic Resonance materials in physics, biology and medicine, 22(1), 3341. 
[95] Davies, N. P., Wilson, M., Natarajan, K., Sun, Y., MacPherson, L., Brundler, M. A., ... $\&$ Peet, A. C. (2010). Non invasive detection of glycine as a biomarker of malignancy in childhood brain tumours using in vivo $1 \mathrm{H}$ MRS at 1.5 Tesla confirmed by ex vivo high resolution magic angle spinning NMR. NMR in biomedicine, 23(1), 80-87.

[96] Prescot, A. P., Wang, L., Brown, J., Jensen, J. E., Kaufman, M. J., \& Renshaw, P. F. (2006). In vivo detection of brain glycine with echo time averaged $1 \mathrm{H}$ magnetic resonance spectroscopy at 4.0 T. Magnetic resonance in medicine, 55(3), 681-686

[97] Gambarota, G., Mekle, R., Xin, L., Hergt, M., van der Zwaag, W., Krueger, G., \& Gruetter, R. (2009). In vivo measurement of glycine with short echo-time 1H MRS in human brain at $7 \mathrm{~T}$. Magnetic resonance materials in physics, biology and medicine, 22(1), 1-4.

[98] Gambarota, G., Xin, L., Perazzolo, C., Kohler, I., Mlynárik, V., \& Gruetter, R. (2008). In vivo $1 \mathrm{H}$ NMR measurement of glycine in rat brain at $9.4 \mathrm{~T}$ at short echo time. Magnetic resonance in medicine, 60(3), 727-731.

[99] Mulkern, R. V., Bowers, J. L., Peled, S., Kraft, R. A., \& Williamson, D. S. (1996). Citrate signal enhancement with a homonuclear $\mathbf{J}$ refocusing modification to double echo PRESS sequences. Magnetic resonance in medicine, 36(5), 775-780.

[100] Gambarota, G., Van Der Graaf, M., Klomp, D., Mulkern, R. V., \& Heerschap, A. (2005). Echo-time independent signal modulations using PRESS sequences: a new approach to spectral editing of strongly coupled AB spin systems. Journal of magnetic resonance, 177(2), 299-306.

[101] Kickler, N., Gambarota, G., Mekle, R., Gruetter, R., \& Mulkern, R. (2010). Echo-time independent signal modulations for strongly coupled systems in triple echo localization schemes: an extension of S-PRESS editing. Journal of magnetic resonance, 203(1), 108-112.

[102] Pople, J. A., Schneider, W. G., \& Bernstein, H. J. (1959). High-resolution nuclear magnetic resonance. McGraw-Hill Inc., USA.

[103] Corio, P. L. (1966). Structure of high-resolution NMR spectra. Academic Press, New York.

[104] Abragam, A. (1961). The principles of nuclear magnetism. Oxford University Press, London.

[105] Canet, D. (1996). Nuclear magnetic resonance: concepts and methods. Wiley, New York, USA.

[106] Becker, E. D. (1999). High resolution NMR: theory and chemical applications. Academic Press. San Diego, USA. 
[107] Goldman, M. (1988) Quantum description of high-resolution NMR in liquids. Oxford University Press, Oxford.

[108] Mulkern, R., \& Bowers, J. (1994). Density matrix calculations of AB spectra from multipulse sequences: quantum mechanics meets in vivo spectroscopy. Concepts in magnetic resonance, 6(1), 1-23.

[109] Corio, P. L. (1960). The analysis of nuclear magnetic resonance spectra. Chemical reviews, 60(4), 363-429.

[110] Lynden-Bell, R. M. (1967). The calculation of line shapes by density matrix methods. Progress in nuclear magnetic resonance spectroscopy, 2, 163-204.

[111] Farrar, T. C. (1990). Density matrices in NMR spectroscopy: part I. Concepts in magnetic resonance, 2(1), 1-12.

[112] Farrar, T. C. (1990). Density matrices in NMR spectroscopy: part II. Concepts in magnetic resonance, 2(1), 55-61.

[113] Smith, S. A., Palke, W. E., \& Gerig, J. T. (1992). The Hamiltonians of NMR. part I. Concepts in magnetic resonance, 4(2), 107-144.

[114] Smith, S. A., Palke, W. E., \& Gerig, J. T. (1992). The Hamiltonians of NMR. Part II. Concepts in magnetic resonance, 4(3), 181-204.

[115] Smith, S. A., Palke, W. E., \& Gerig, J. T. (1993). The Hamiltonians of NMR. Part III. Concepts in magnetic resonance, 5(2), 151-177.

[116] Smith, S. A., Levante, T. O., Meier, B. H., \& Ernst, R. R. (1994). Computer simulations in magnetic resonance. An object-oriented programming approach. Journal of magnetic resonance, Series A, 106(1), 75-105.

[117] Buckley, P. D., Jolley, K. W., \& Pinder, D. N. (1975). Application of density matrix theory to NMR line-shape calculations. Progress in nuclear magnetic resonance spectroscopy, $10(1), 1-26$.

[118] Slotboom, J., Mehlkopf, A. F., \& Bovee, W. M. M. J. (1994). The Bloch equations for an $\mathrm{AB}$ system and the design of spin-state-selective RF pulses for coupled spin systems. Journal of magnetic resonance, Series A, 106(2), 159-167.

[119] Hennig, J., Thiel, T., \& Speck, O. (1997). Improved sensitivity to overlapping multiplet signals in in vivo proton spectroscopy using a multiecho volume selective (CPRESS) experiment. Magnetic resonance in medicine, 37(6), 816-820. 
[120] Soher, B. J., Pattany, P. M., Matson, G. B., \& Maudsley, A. A. (2005). Observation of coupled $1 \mathrm{H}$ metabolite resonances at long TE. Magnetic resonance in medicine, 53(6), 1283 1287. 


\section{Figure Legends}

Figure 1. Bust of Janus, The Vatican Museums, Rome.

Figure 2. The energy level diagram for a single spin (left panel) and a two-spin system (uncoupled spins, center panel; coupled spins, right panel). The J couling interaction modifies the energy levels with the effect of splitting the single peak into a doublet. The dotted lines (center and right panel) indicate the energy levels of the uncoupled spins, to aid visualization of the $\mathrm{J}$ coupling effects in the right panel. The allowed transitions are indicated by the arrows.

Figure 3. ${ }^{1} \mathrm{H}$ MR spectra of citrate acquired at $3 \mathrm{~T}$ using a PRESS sequence with three refocusing $180^{\circ}$ pulses. The total echo time is equal to $80 \mathrm{~ms}$. The MR spectrum obtained by density matrix simulation is in excellent agreement with the experimental spectrum and with the spectrum obtained by analytical calculations. The experimental data were acquired on a phantom containing citrate and creatine.

Figure 4. ${ }^{1} \mathrm{H}$ MR spectra of citrate acquired at $3 \mathrm{~T}$ using a PRESS sequence with three refocusing $180^{\circ}$ pulses. The simulated MR spectrum is in excellent agreement with the experimental spectrum and with the spectrum obtained by analytical calculations. The total echo time is the same as that of the MR spectra shown in Figure 3. The interpulse delays, on the other hand, are different than those of Figure 3.

Figure 5. PRESS signal intensity of citrate at $3 \mathrm{~T}$ as a function of $\mathrm{TE}_{1}$ and $\mathrm{TE}_{2}$ (total echo time $\mathrm{TE}=\mathrm{TE}_{1}+\mathrm{TE}_{2}$ ). Two different representations of the signal intensity are shown: 3D graph (right) and a flat surface graph (left). The latter representation allows for improved visualization of the optimal interpulses that maximize the signal intensity.

Figure 6. PRESS signal intensity of citrate at $11.7 \mathrm{~T}$ as a function of $\mathrm{TE}_{1}$ and $\mathrm{TE}_{2}$ (total echo time $\mathrm{TE}=\mathrm{TE}_{1}+\mathrm{TE}_{2}$ ). As in Figure 5, two different representations of the signal intensity are shown: 3D graph (right) and a flat surface graph (left). The spin system is approaching the behaviour of weakly coupled system; however, the 'wiggles' along the signal intensity 'waves' indicate that strongly coupled effects are still present. 


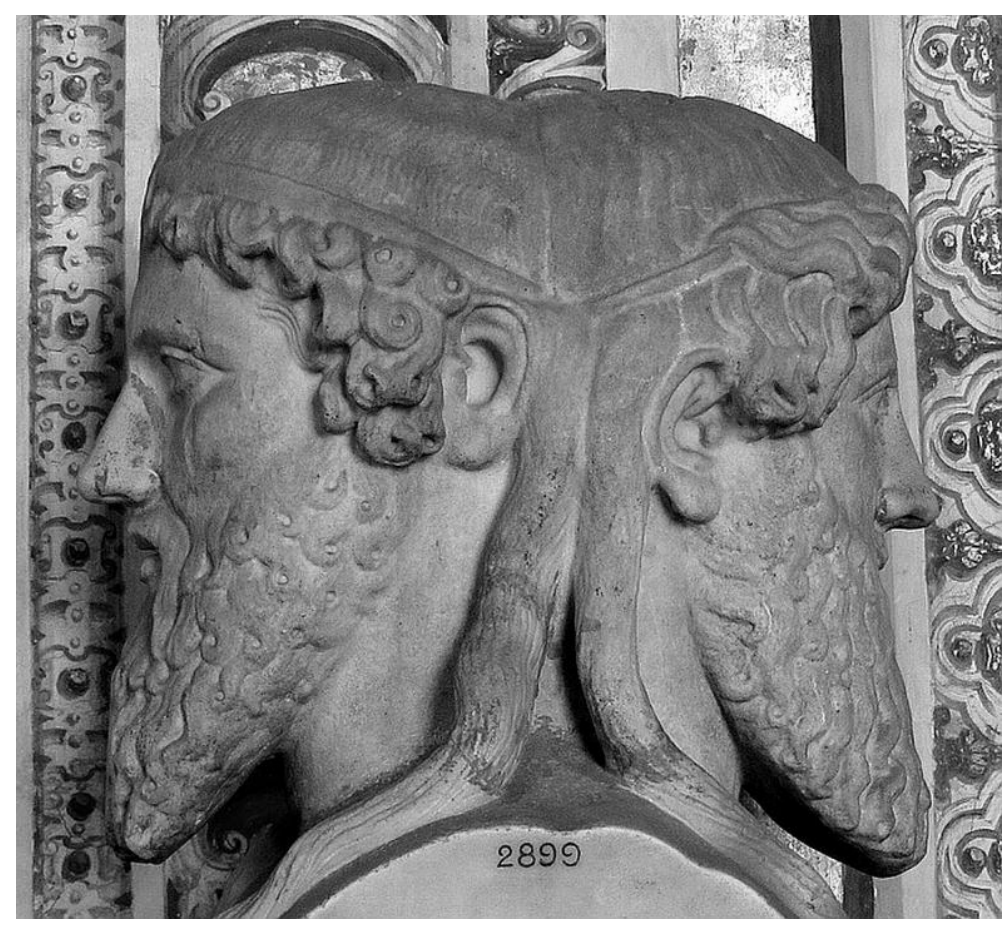

Figure 1. Bust of Janus, The Vatican Museums, Rome. 

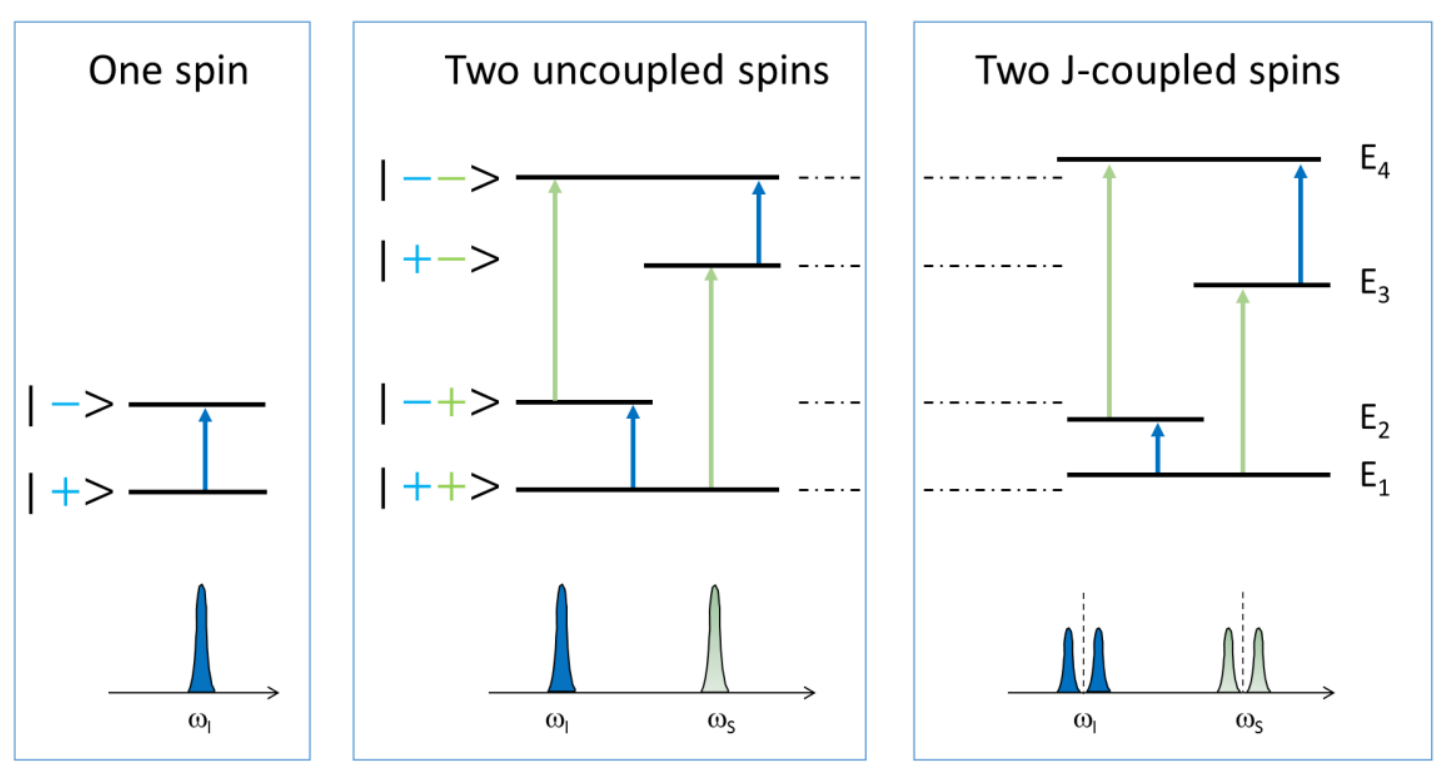

Figure 2. The energy level diagram for a single spin (left panel) and a two-spin system (uncoupled spins, center panel; coupled spins, right panel). The J couling interaction modifies the energy levels with the effect of splitting the single peak into a doublet. The dotted lines (center and right panel) indicate the energy levels of the uncoupled spins, to aid visualization of the $\mathrm{J}$ coupling effects in the right panel. The allowed transitions are indicated by the arrows. 


$$
\mathrm{TE}=80 \mathrm{~ms}
$$

\section{Experiment}

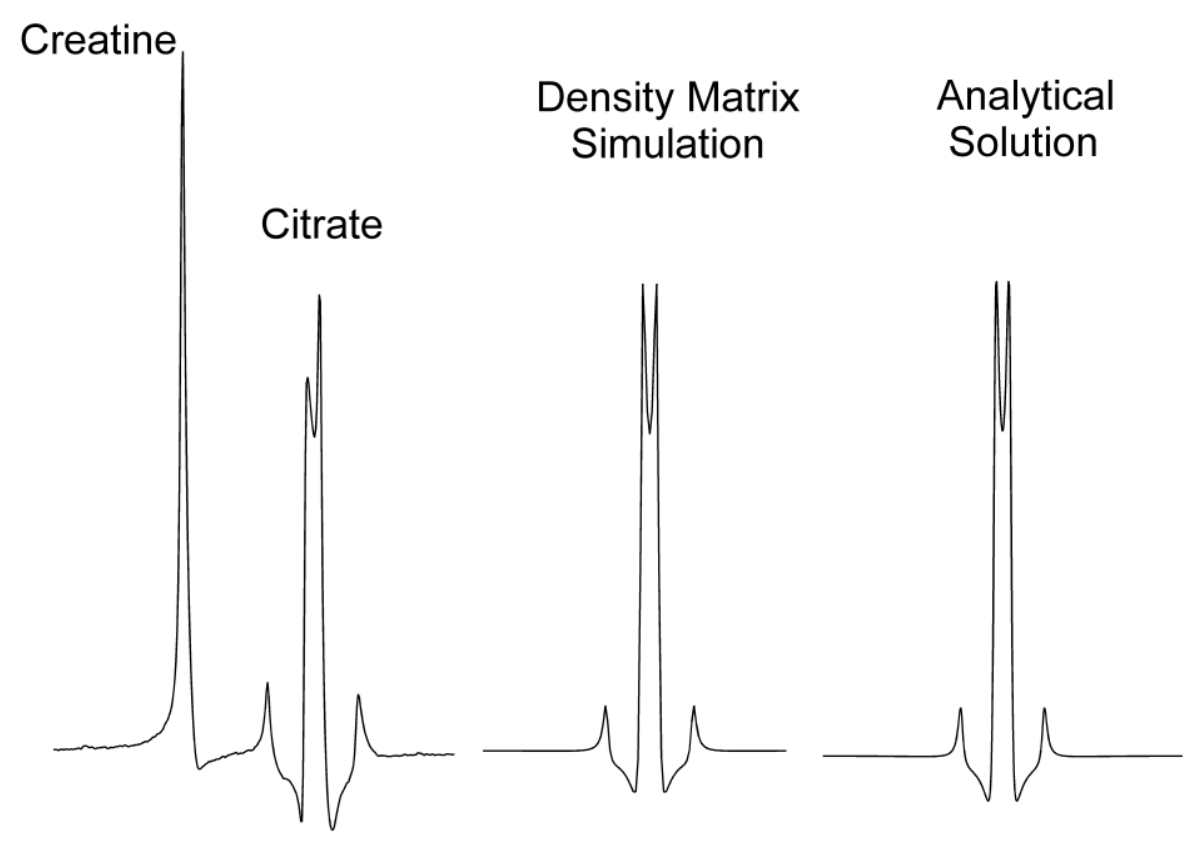

Figure 3. ${ }^{1} \mathrm{H}$ MR spectra of citrate acquired at $3 \mathrm{~T}$ using a PRESS sequence with three refocusing $180^{\circ}$ pulses. The total echo time is equal to $80 \mathrm{~ms}$. The MR spectrum obtained by density matrix simulation is in excellent agreement with the experimental spectrum and with the spectrum obtained by analytical calculations. The experimental data were acquired on a phantom containing citrate and creatine. 


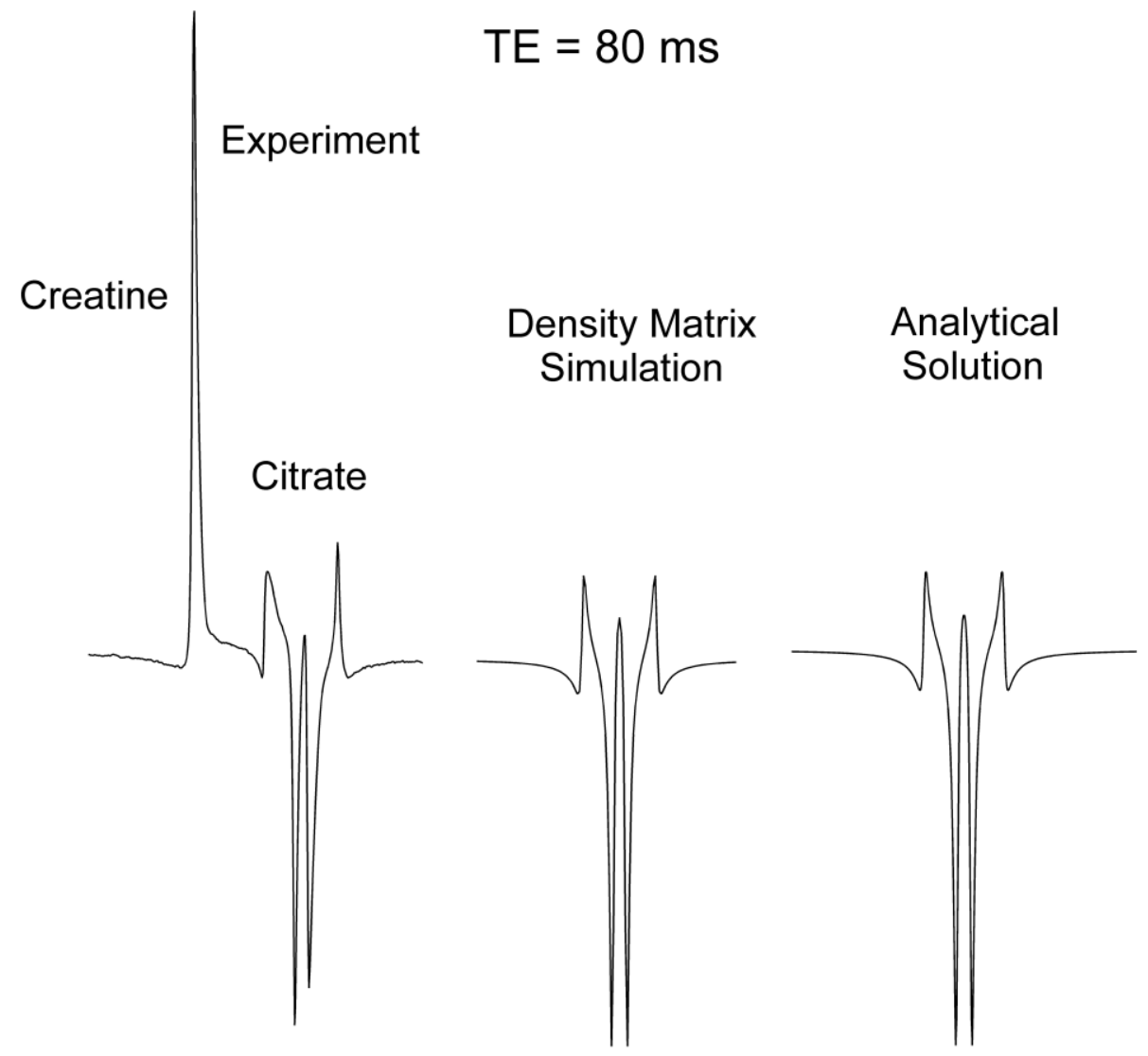

Figure 4. ${ }^{1} \mathrm{H}$ MR spectra of citrate acquired at $3 \mathrm{~T}$ using a PRESS sequence with three refocusing $180^{\circ}$ pulses. The simulated MR spectrum is in excellent agreement with the experimental spectrum and with the spectrum obtained by analytical calculations. The total echo time is the same as that of the MR spectra shown in Figure 3. The interpulse delays, on the other hand, are different than those of Figure 3. 

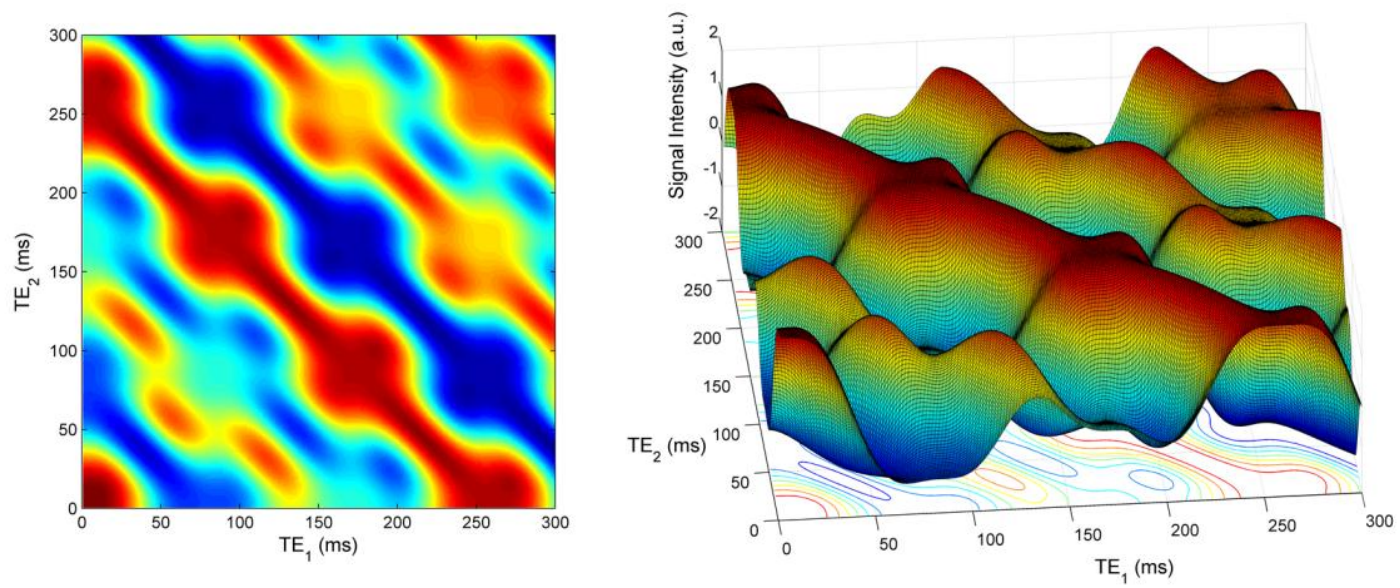

Figure 5. PRESS signal intensity of citrate at $3 \mathrm{~T}$ as a function of $\mathrm{TE}_{1}$ and $\mathrm{TE}_{2}$ (total echo time $\mathrm{TE}=\mathrm{TE}_{1}+\mathrm{TE}_{2}$ ). Two different representations of the signal intensity are shown: 3D graph (right) and a flat surface graph (left). The latter representation allows for improved visualization of the optimal interpulses that maximize the signal intensity. 

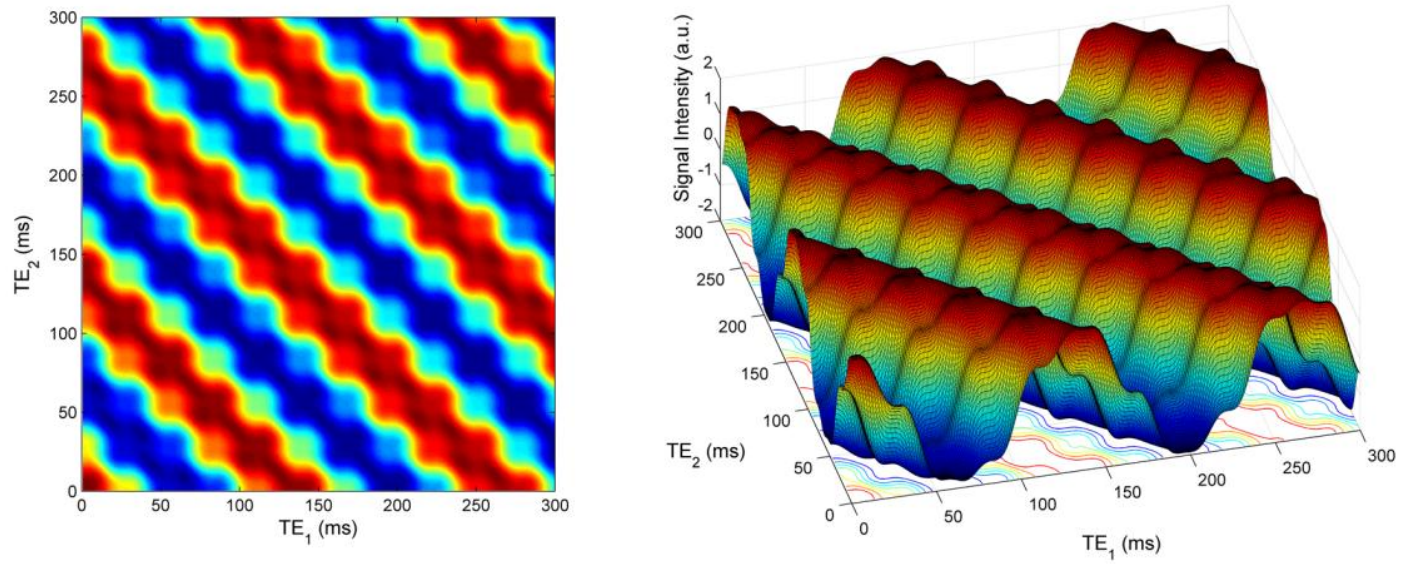

Figure 6. PRESS signal intensity of citrate at $11.7 \mathrm{~T}$ as a function of $\mathrm{TE}_{1}$ and $\mathrm{TE}_{2}$ (total echo time $\mathrm{TE}=\mathrm{TE}_{1}+\mathrm{TE}_{2}$ ). As in Figure 5, two different representations of the signal intensity are shown: 3D graph (right) and a flat surface graph (left). The spin system is approaching the behaviour of weakly coupled system; however, the 'wiggles' along the signal intensity 'waves' indicate that strongly coupled effects are still present. 